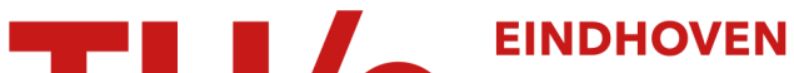 \\ UNIVERSITY OF \\ TECHNOLOGY
}

\section{Uniform asymptotic theory of edge diffraction}

Citation for published version (APA):

Lewis, R. M., \& Boersma, J. (1969). Uniform asymptotic theory of edge diffraction. Journal of Mathematical Physics, 10(12), 2291-2305. https://doi.org/10.1063/1.1664835

DOI:

10.1063/1.1664835

Document status and date:

Published: 01/01/1969

\section{Document Version:}

Publisher's PDF, also known as Version of Record (includes final page, issue and volume numbers)

\section{Please check the document version of this publication:}

- A submitted manuscript is the version of the article upon submission and before peer-review. There can be important differences between the submitted version and the official published version of record. People interested in the research are advised to contact the author for the final version of the publication, or visit the $\mathrm{DOI}$ to the publisher's website.

- The final author version and the galley proof are versions of the publication after peer review.

- The final published version features the final layout of the paper including the volume, issue and page numbers.

Link to publication

\section{General rights}

Copyright and moral rights for the publications made accessible in the public portal are retained by the authors and/or other copyright owners and it is a condition of accessing publications that users recognise and abide by the legal requirements associated with these rights.

- Users may download and print one copy of any publication from the public portal for the purpose of private study or research.

- You may not further distribute the material or use it for any profit-making activity or commercial gain

- You may freely distribute the URL identifying the publication in the public portal.

If the publication is distributed under the terms of Article $25 \mathrm{fa}$ of the Dutch Copyright Act, indicated by the "Taverne" license above, please follow below link for the End User Agreement:

www.tue.nl/taverne

Take down policy

If you believe that this document breaches copyright please contact us at:

openaccess@tue.nl

providing details and we will investigate your claim. 


\title{
Uniform Asymptotic Theory of Edge Diffraction*
}

\author{
Robert M. Lewis $\dagger$ AND Johannes Boersma \\ Courant Institute of Mathematical Sciences, New York University \\ and \\ Department of Mathematics, Technological University, Eindhoven,The Netherlands
}

\author{
(Received 28 September 1966)
}

\begin{abstract}
Geometrical optics fails to account for the phenomenon of diffraction, i.e., the existence of nonzero fields in the geometrical shadow. Keller's geometrical theory of diffraction accounts for this phenomenon by providing correction terms to the geometrical optics field, in the form of a high-frequency asymptotic expansion. In problems involving screens with apertures, this asymptotic expansion fails at the edge of the screen and on shadow boundaries where the expansion has singularities. The uniform asymptotic theory presented here provides a new asymptotic solution of the diffraction problem which is uniformly valid near edges and shadow boundaries. Away from these regions the solution reduces to that of Keller's theory. However, singularities at any caustics other than the edge are not corrected.
\end{abstract}

\section{INTRODUCTION}

Geometrical optics fails to account for the phenomenon of diffraction, i.e., the existence of nonzero fields in the geometrical shadow. It is now known that the geometrical-optics field corresponds to the leading term of a high-frequency asymptotic expansion of the solution of a boundary-value problem for the reduced wave equation or Maxwell's equations, and that higherorder terms account for diffraction. Keller's "geometrical theory of diffraction" ${ }^{1.2}$ provides a systematic means for computing these terms.

In this paper we consider problems of diffraction by screens. The screens may be portions of planes or other smooth surfaces bounded by smooth curves, and the prescribed incident wave may be arbitrary. We consider here only scalar problems for the reduced wave equation with boundary conditions of the first or second kind ( $u=0$ or $\partial u / \partial n=0)$ on the screen. In Sec. 2 we present a brief but self-contained treatment of Keller's geometrical theory for such problems. This theory depends on a "diffraction coefficient" the value of which is obtained from a special ("canonical") problem, the problem of diffraction of a plane wave by a half plane. Sommerfeld's solution of this problem is discussed in Sec. 3 and there the diffraction coefficient is evaluated.

The geometrical theory has several shortcomings. It fails at the shadow boundaries of the incident and reflected waves as well as at the edge of the screen where the "diffracted wave" becomes infinite. Furthermore, it is difficult to justify the determination of the

\footnotetext{
* The research in this paper was supported by the Air Force Cambridge Research Laboratories, Office of Aerospace Research, under Contract No. AF 19(628)3868. Reproduction in whole or in part is permitted for any purpose of the U.S. Government.

+ R. M. Lewis died on 7 November 1968 .

1 J. B. Keller, J. Opt. Soc. Am. 52, 116 (1962).

2 R. M. Lewis and J. B. Keller, New York University Research Report EM-194, 1964.
}

diffraction coefficient by comparison with the solution of the canonical problem, and this procedure cannot be generalized to yield higher-order terms in the diffracted field. These shortcomings are overcome by the method presented in Secs. 4 and 5 of this paper. Other shortcomings of the geometrical theory (the failure at caustics of the problem) remain. Like Keller's theory, ours is formal in the sense that we do not rigorously prove the asymptotic nature of the solution obtained.

Our approach is motivated by a new representation of the solution of the half-plane problem. By using simple concepts of the geometrical theory such as incident-, reflected-, and diffracted-phase functions, we show in Sec. 3 that Sommerfeld's solution can be expressed in a remarkably simple and suggestive form. This representation involves a special function $f$ which is discussed briefly in Appendix A. It is closely related to the Fresnel integral functions.

The geometrical theory of diffraction is based on an "ansatz" in the form of an asymptotic series involving certain "phase" and "amplitude" functions. By inserting the series into the reduced wave equation, one obtains the eikonal equation for the phase function $s(\mathbf{x})$ and a sequence of transport equations for the amplitude functions $z_{m}(\mathbf{x})$. These equations can be solved by introducing lines in $\mathbf{x}$-space called "rays." Our approach is based on a new ansatz that involves the function $f$. Away from the edge of the screen and the shadow boundaries, the new expression reduces to one of the same form as Keller used. Therefore the phase and amplitude functions which appear in the new ansatz also satisfy the eikonal and transport equations. In Keller's theory there is an undetermined "initial condition" for the transport equation of order zero. This leads to the diffraction coefficient. In our approach the initial condition is 
uniquely determined by imposing the "edge condition," which is a part of the rigorous formulation of the boundary-value problem. Away from the edge and shadow boundaries, the leading term of our result reduces to Keller's, and we verify his expression for the diffraction coefficient.

By construction our solution is continuous and finite at the edge of the screen because the edge condition demands this. It is not immediately obvious that it is also continuous at the shadow boundaries. However, in Sec. 4 we compute the leading term of our expansion and prove that it is continuous at the shadow boundaries as well as at the edge. (For this reason we call our asymptotic solution "uniform.") The generalization of this theorem to higher-order terms has not yet been proved. In Sec. 5 we compute the next term of our expansion. In order to simplify the calculations we restrict the problem at this point to screens which are portions of planes. The computation requires an expression for the Laplacian in "ray coordinates" which are not orthogonal. This expression is derived in Appendix C. When our result is evaluated away from the edge and shadow boundaries, it again reduces to an expression of the form used in the geometrical theory, but now the first two terms of the diffracted wave are given. The second term can be expressed in a form that involves Keller's diffraction coefficient and a new coefficient. There is a special problem (grazing incidence with boundary condition $\partial u / \partial n=0)$ in which Keller's diffraction coefficient vanishes and the second term becomes important. For this case Keller has obtained a special diffraction coefficient by using a special canonical problem. In this case our new coefficient reduces to his.

In several respects our theory is incomplete. We have already mentioned the unproved conjecture that all terms are continuous at the shadow boundary. There is a second unproved conjecture: We have obtained the first two terms of the expansion, at least for plane screens. (This is probably not an essential restriction.) It seems likely that the procedure can be continued to yield higher-order terms. But this too is not obvious and has not yet been proved. [Note added in proof: Both conjectures were proved recently; see D. S. Ahluwalia, R. M. Lewis, and J. Boersma, SIAM J. Appl. Math. 16, 703 (1968).] Furthermore, as we have mentioned, our theory also fails at caustics of the incident and reflected waves and caustic points of the diffracted wave other than those on the edge.

Our theory is also incomplete in another sense. For nonplanar screens, diffracted rays emanating from the edge may strike another portion of the screen giving rise to secondary reflected waves or creeping waves. Such waves are not included in our theory. (See the remarks at the end of Sec. 4.)

Uniform expansions which are valid at caustics have recently been obtained by Kravtsov ${ }^{3}$ and Ludwig. ${ }^{4}$ In fact, their work partially motivated our approach to the problem of diffraction by screens. A second motivation came from the work of Lewis ${ }^{5}$ on the uniform transition from the "forerunner" to the "main signal" of a transient wave propagating into a dispersive medium.

The main motivation, however, came from the recent work of Wolfe. ${ }^{6}$ Wolfe considered some special cases of the problems treated here, involving plane and spherical waves incident on a screen which is a portion of a plane. For these problems he obtained uniform asymptotic solutions by means of an ansatz involving Fresnel integrals. This ansatz, which was given in terms of ray coordinates, was substituted into the reduced wave equation which had to be transformed to these same coordinates. This obscures several important features of the method. For example, one does not see that the ansatz involves functions that are identical to the phase and amplitude functions of the geometrical theory. As a consequence Wolfe's method is more complicated than ours. In addition, Wolfe relies on the use of the canonical half-plane problem, since the Fresnel-integral part of his ansatz is derived from the uniform asymptotic expansion of the solution of the half-plane problem for the same incident wave. Since this problem has been solved only for special incident waves (plane, cylindrical, and spherical), this restricts the generality of his method. Nevertheless the essential features of our approach are contained in Wolfe's work and we are very much indebted to him. We are of course also greatly indebted to Keller, not only for his geometrical theory of diffraction, but also for his continuing interest and advice in the course of Wolfe's work and our own.

In closing this introduction we wish to mention some problems closely related to the one treated here. The problem of diffraction by a screen is a special case of diffraction by objects which are locally wedgeshaped. (Along the edge the screen is locally a zeroangled wedge.) Such problems can be treated by Keller's theory. The generalization of our method to these problems is currently under consideration. There

\footnotetext{
${ }^{3}$ Yu. A. Kravtsov, Radiofiz. 7, 664 (1964).

4 D. Ludwig, Commun. Pure Appl. Math. 19, 215 (1966).

5 R. M. Lewis, Proceedings of the U.R.S.I. Symposium on Electromagnetic Wave Theory (Delft, The Netherlands, 1965).

"P. Wolfe, "Diffraction of a Scalar Wave by a Plane Screen," Ph.D. thesis, New York University, 1965.
} 
is also a fairly obvious generalization of our approach to problems of diffraction by screens in inhomogeneous media. We have not included a treatment of such problems because the added complications are not justified by the practical importance of the generalization. In addition it is fairly clear that the method presented here can be applied to Maxwell's equations and other linear partial-differential equations, but this has not yet been done. Many diffraction problems (e.g., diffraction by a slit or by a circular aperture in a plane screen) involve "multiple diffraction" (waves produced at one edge are incident on another). Such problems have been treated by Keller and will be treated by our method in a forthcoming sequel to this paper. Finally there is a whole class of problems of diffraction by smooth objects that can be treated by another part of Keller's theory. Recently uniform asymptotic solutions of these problems have been obtained. ${ }^{7.8}$ These solutions improve on Keller's theory in much the same way as the method presented here improves on his theory of edge diffraction.

\section{KELLER'S GEOMETRICAL THEORY OF DIFFRACTION}

In this section we present a summary of that part of Keller's theory which relates to diffraction by an edge of a screen. Further details are given in References 1 and 2. It is important for us to summarize Keller's theory not only because our work was motivated by it, but because we make heavy use of his results. In Secs. 4 and 5, we use almost all the equations derived here.

We consider asymptotic solutions of the reduced wave equation

of the form

$$
\Delta u+k^{2} u=0
$$

$$
u \sim e^{i k s(\mathbf{x})} \sum_{m=0}^{\infty}(i k)^{-m} z_{m}(\mathbf{x}), \quad k \rightarrow \infty .
$$

By inserting (2.2) into (2.1), we find that the phase function $s(\mathbf{x})$ satisfies the eikonal equation of geometrical optics

$$
(\nabla s)^{2}=1,
$$

while the amplitude functions $z_{m}(\mathbf{x})$ satisfy the recursive system of transport equations

$$
\begin{array}{r}
2 \nabla s \cdot \nabla z_{m}+z_{m} \Delta s=-\Delta z_{m-1} ; \quad m=0,1,2, \cdots, \\
z_{-1} \equiv 0 .
\end{array}
$$

Solution of (2.3) may be described as follows: Given a surface (wavefront) on which $s$ has the constant value

\footnotetext{
${ }^{7}$ R. M. Lewis, N. Bleistein, and D. Ludwig, Commun. Pure Appl. Math. 20, 295 (1967).

${ }^{8}$ D. Ludwig, Commun. Pure Appl. Math. 20, 103 (1967).
}

$s_{0}$, we introduce the two-parameter family of straight lines (rays) orthogonal to the surface. If $\sigma$ denotes distance along the rays from the wavefront (measured positively in the direction of increasing $s$ ), then on each ray

$$
s=s_{0}+\sigma .
$$

It is then clear that (2.5) satisfies (2.3).

Let $\sigma_{2}$ and $\sigma_{3}$ be the two parameters that label the rays and let us describe a ray parametrically in the form

$$
\mathbf{x}=\mathbf{x}(\sigma)=\mathbf{x}\left(\sigma, \sigma_{2}, \sigma_{3}\right) .
$$

If we set $\sigma=\sigma_{1}$, then (2.6) defines a transformation from $\left(\sigma_{1}, \sigma_{2}, \sigma_{3}\right)$-space to $\left(x_{1}, x_{2}, x_{3}\right)$-space and the Jacobian of the transformation is

$$
j=j(\sigma)=j\left(\sigma, \sigma_{2}, \sigma_{3}\right)=\operatorname{det}\left(\frac{\partial x_{i}}{\partial \sigma_{j}}\right), \quad i, j=1,2,3 .
$$

For given $z_{m-1}$ it is easy to see that (2.4) is an ordinary differential equation for $z_{m}$ along a ray. The solution can be expressed in the form

$$
\begin{array}{r}
z_{m}(\sigma)=\left|\frac{j\left(\sigma_{0}\right)}{j(\sigma)}\right|^{\frac{1}{2}} z_{m}\left(\sigma_{0}\right)-\frac{1}{2} \int_{\sigma_{0}}^{\sigma}\left|\frac{j\left(\sigma^{\prime}\right)}{j(\sigma)}\right|^{\frac{1}{2}} \Delta z_{m-1}\left(\sigma^{\prime}\right) d \sigma^{\prime}, \\
m=0,1,2, \cdots .
\end{array}
$$

Here $z_{m}(\sigma)=z_{m}\left[\mathbf{x}\left(\sigma, \sigma_{2}, \sigma_{3}\right)\right]$ is the value of $z_{m}$ at a point $\sigma$ on a given ray. The solution (2.8) is given in terms of an "initial value" $z_{m}\left(\sigma_{0}\right)$ at some fixed point on each ray. For $m=0$ we note that the second term of (2.8) is absent because $z_{-1} \equiv 0$. Two alternative expressions for the ratio of Jacobians are sometimes useful:

$$
\frac{j\left(\sigma_{0}\right)}{j(\sigma)}=\frac{d a\left(\sigma_{0}\right)}{d a(\sigma)}=\frac{\left(\rho_{2}+\sigma_{0}\right)\left(\rho_{3}+\sigma_{0}\right)}{\left(\rho_{2}+\sigma\right)\left(\rho_{3}+\sigma\right)} .
$$

Here $d a(\sigma)$ is the cross-sectional area of an infinitesimal tube of rays, while $\rho_{2}\left(\sigma_{2}, \sigma_{3}\right)$ and $\rho_{3}\left(\sigma_{2}, \sigma_{3}\right)$ are the principal radii of curvature of the wavefront $\sigma=0$.

At the two points $\sigma=-\rho_{2}$ and $\sigma=-\rho_{3}$ on each ray, we see from (2.9) that $z_{m}$ becomes infinite and the integral in (2.8) will, in general, diverge. Such points are called caustic points. They lie on the caustic, which is, in general, a two-sheeted surface forming the envelope of the family of rays (the rays are tangent to the caustic). We shall require an alternative form of (2.8) which remains valid when $\sigma_{0}=0$ is a caustic point. First we rewrite (2.8) in the form

$$
\begin{aligned}
|j(\sigma)|^{\frac{1}{2}} z_{m}(\sigma)=\mid & \left|j\left(\sigma_{0}\right)\right|^{\frac{1}{2}} z_{m}\left(\sigma_{0}\right) \\
& \quad-\frac{1}{2} \int_{\sigma_{0}}^{\sigma}\left|j\left(\sigma^{\prime}\right)\right|^{\frac{1}{2}} \Delta z_{m-1}\left(\sigma^{\prime}\right) d \sigma^{\prime} .
\end{aligned}
$$


Then we express the integral in (2.10) in the form

$$
\int_{\sigma_{0}}^{\sigma}=f_{0}^{\sigma}-f_{0}^{\sigma_{0}} \text {. }
$$

Here the dash denotes the "finite part" 9 of a divergent integral. (The ordinary integrals would diverge at $\sigma^{\prime}=0$.) Now (2.10) becomes

$|j(\sigma)|^{\frac{1}{2}} z_{m}(\sigma)+\frac{1}{2} f_{0}^{\sigma}=\left|j\left(\sigma_{0}\right)\right|^{\frac{1}{2}} z_{m}\left(\sigma_{0}\right)+\frac{1}{2} f_{0}^{\sigma_{0}}$.

The right side of (2.12) is independent of $\sigma$. If we denote its value by $\zeta_{m}$, then we obtain

$$
\begin{gathered}
z_{\dot{m}}(\sigma)=\frac{\zeta_{m}}{|j(\sigma)|^{\frac{1}{2}}}-\frac{1}{2} f_{0}^{\sigma}\left|\frac{j\left(\sigma^{\prime}\right)}{j(\sigma)}\right|^{\frac{1}{2}} \Delta z_{m-1}\left(\sigma^{\prime}\right) d \sigma^{\prime}, \\
m=0,1,2, \cdots
\end{gathered}
$$

This is the required modification of (2.8). The "initial value" $\zeta_{m}\left(\sigma_{2}, \sigma_{3}\right)$ has first to be determined before (2.13) is useful. We will see in Sec. 5 that the finitepart integrals are a useful computational tool. For $m=0$, the integral in (2.13) is again absent.

We now consider the problem of diffraction by a screen $S$. The screen is a portion of a smooth surface. It is bounded by an edge $E$ consisting of a smooth curve

$$
\mathbf{x}=\mathbf{x}_{0}(\eta)
$$

Here $\eta$ is an arclength parameter. For example, $S$ might be an infinite plane with a circular aperture or it could be the complementary disk. Alternatively the aperture may have any smooth shape. In general, $S$ need not be a portion of a plane. We consider an incident wave

$$
u_{0}^{\mathrm{i}} \sim e^{i k s} \sum_{m=0}^{\infty}(i k)^{-m} z_{m}^{\mathrm{i}},
$$

which is an asymptotic solution of (2.1). Then $s^{i}$ and the $z_{m}^{\mathrm{i}}$ satisfy the equations derived above. The total field $u$ is a solution of (2.1) and satisfies a boundary condition on the screen. We shall consider simultaneously the two conditions

and

$$
u=0 \quad \text { on } S
$$

$$
\frac{\partial u}{\partial n}=\mathbf{N} \cdot \nabla u=0 \quad \text { on } S \text {. }
$$

Here $\mathbf{N}$ is a unit normal vector on $S$. In addition, $u-u_{0}^{\mathrm{i}}$ is required to be "outgoing."

To solve the diffraction problem, we first set

\footnotetext{
${ }^{9}$ Let $f(\epsilon)=\int_{\epsilon}^{b} g(x) d x$ have an asymptotic expansion in (perhaps fractional) powers of $\epsilon$ for $\epsilon \rightarrow 0$. The coefficient of $\epsilon^{0}=1$ in the expansion is called the finite part of the integral and will be denoted by $f_{0}^{b} g(x) d x$.
}

$u=u_{0}^{\mathrm{i}}+u_{0}^{\mathrm{r}}$. We assume that the reflected wave $u_{0}^{\mathrm{r}}$ has an asymptotic expansion

$$
u_{0}^{\mathrm{r}} \sim e^{i k s^{\mathrm{r}}} \sum_{m=0}^{\infty}(i k)^{-m} z_{m}^{\mathrm{r}} .
$$

Then (2.16) will be satisfied, provided

$$
s^{r}=s^{\mathrm{i}} \text { on } S
$$

and, for the boundary condition $u=0$,

$$
z_{m}^{\mathbf{r}}=-z_{m}^{\mathrm{i}} \quad \text { on } S ; \quad m=0,1,2, \cdots .
$$

For the case $\partial u / \partial n=0,(2.19)$ is replaced by

$$
\begin{array}{r}
z_{m}^{\mathrm{i}} \frac{\partial s^{\mathrm{i}}}{\partial n}+z_{m}^{\mathrm{r}} \frac{\partial s^{\mathrm{r}}}{\partial n}+\frac{\partial z_{m-1}^{\mathrm{i}}}{\partial n}+\frac{\partial z_{m-1}^{\mathrm{r}}}{\partial n}=0 \quad \text { on } S, \\
m=0,1,2, \cdots
\end{array}
$$

It can be shown that (2.18) implies that the incident and reflected rays (which have the direction $\nabla s^{i}$ and $\nabla S^{\mathrm{I}}$, respectively) satisfy the law of reflection of geometrical optics. If $\psi$ is the angle of incidence (= angle of reflection), then $\partial s^{\mathrm{T}} / \partial n=\cos \psi=-\partial s^{\mathrm{i}} / \partial n$ and $(2.20)$ becomes

$$
\begin{array}{r}
z_{m}^{\mathrm{r}}=z_{m}^{\mathrm{i}}-\frac{1}{\cos \psi}\left(\frac{\partial z_{m-1}^{\mathrm{i}}}{\partial n}+\frac{\partial z_{m-1}^{\mathrm{r}}}{\partial n}\right) \text { on } S \\
m=0,1,2, \cdots
\end{array}
$$

Thus $s^{\mathrm{r}}$ is determined on the reflected rays by (2.18) and (2.5), while the functions $z_{m}^{\mathrm{r}}$ are given by (2.8) with $z_{m}\left(\sigma_{0}\right)$ determined by (2.19) or (2.21). We note that both $u_{0}^{\mathrm{i}}$ and $u_{0}^{\mathrm{r}}$ are zero in their respective "shadow regions," i.e., where there are no incident or reflected rays. Thus each has an "illuminated region" separated from the corresponding shadow region by a shadow boundary surface.

The leading term $u=u_{0}^{\mathrm{i}}+u_{0}^{\mathrm{r}} \sim z_{0}^{\mathrm{i}} \exp \left(i k s^{\mathrm{i}}\right)+$ $z_{0}^{r} \exp \left(i k s^{r}\right)$ is the geometrical-optics solution of the problem, which of course fails to account for diffraction phenomena (nonzero fields in the shadows). The full solution $(2.15)+(2.17)$ is correct only to first order because, according to Keller's theory, there is an additional diffracted wave $\hat{u}$. Then

where

$$
u=u_{0}^{\mathrm{i}}+u_{0}^{\mathrm{r}}+\hat{u},
$$

$$
\hat{u} \sim k^{-\frac{1}{2}} e^{i k \hat{s}} \sum_{m=0}^{\infty}(i k)^{-m} \hat{z}_{m}
$$

Of course $\hat{s}$ and the $\hat{z}_{m}$ satisfy the equations derived earlier for phase and amplitude functions. The diffracted rays associated with $\hat{s}$ emanate from the edge $E$ of the screen and

$$
\hat{s}=s^{\mathrm{i}} \text { on } E .
$$


Let us introduce the unit tangent, normal, and binormal vectors $\mathbf{t}, \mathbf{n}$, and $\mathbf{b}$ of $E$. Then $\mathbf{t}=\dot{\mathbf{x}}_{0}(\eta)$, $b=\mathbf{t} \times \mathbf{n}$, and the equations of Frenet,

$$
\dot{\mathbf{t}}=\kappa \mathbf{n}, \quad \dot{\mathbf{n}}=-\kappa \mathbf{t}+\tau \mathbf{b}, \quad \dot{b}=-\tau \mathbf{n},
$$

are satisfied. Here $\kappa$ is the curvature and $\tau$ is the torsion of $E$. If we differentiate (2.24) with respect to $\eta$, we obtain

$$
\boldsymbol{\nabla} \hat{s} \cdot \mathbf{t}=\nabla s^{\mathbf{i}} \cdot \mathbf{t} .
$$

This implies the law of edge diffraction: The diffracted rays make the same angle with the tangent $t$ to the edge as the incident ray at the point of diffraction. Let $\beta=\beta(\eta)$ be this angle. Then, from each point $\mathbf{x}_{0}(\eta)$ of the edge, the diffracted rays emanating from the point generate a cone of semiangle $\beta$. Thus we have a two-parameter family of diffracted rays

$$
\mathbf{x}=\mathbf{x}_{\mathbf{0}}(\eta)+\sigma \mathbf{U}(\eta, \alpha),
$$

where $\mathbf{U}$ is a unit vector given by

$$
\mathbf{U}=\cos \beta \mathbf{t}+\sin \beta \cos \alpha \mathbf{n}+\sin \beta \sin \alpha \mathbf{b} .
$$

We may calculate the Jacobian

$$
j(\sigma)=j(\sigma, \alpha, \eta)=\frac{\partial\left(x_{1}, x_{2}, x_{3}\right)}{\partial(\sigma, \alpha, \eta)}
$$

of the transformation defined by (2.27), using (2.25). We find that

$$
j=\frac{\partial \mathbf{x}}{\partial \sigma} \cdot \frac{\partial \mathbf{x}}{\partial \alpha} \times \frac{\partial \mathbf{x}}{\partial \eta}=\left(\sin ^{2} \beta\right) \sigma\left(1+\frac{\sigma}{\rho}\right),
$$

where

$$
\rho=-\frac{\sin \beta}{\dot{\beta}(\eta)+\kappa \cos \alpha}=-\frac{\sin ^{2} \beta}{\hat{\beta} \sin \beta+\kappa \cos \delta},
$$

and

$$
\cos \delta=\sin \beta \cos \alpha=\mathbf{U} \cdot \mathbf{n} .
$$

Then, if we set $\delta_{m}(\alpha, \eta)=\zeta_{m} / \sin \beta$, (2.13) yields

$$
\begin{aligned}
\hat{z}_{m}(\sigma)= & \left|\sigma\left(1+\frac{\sigma}{\rho}\right)\right|^{-\frac{1}{2}} \delta_{m} \\
& -\frac{1}{2} f_{0}^{\sigma}\left|\frac{\sigma^{\prime}\left(\rho+\sigma^{\prime}\right)}{\sigma(\rho+\sigma)}\right|^{\frac{1}{2}} \Delta \hat{z}_{m-1}\left(\sigma^{\prime}\right) d \sigma^{\prime} ; \\
& m=0,1,2, \cdots .
\end{aligned}
$$

In particular,

$$
\varepsilon_{0}=\left|\sigma\left(1+\frac{\sigma}{\rho}\right)\right|^{-\frac{1}{2}} \delta_{0} .
$$

The undetermined factor $\delta_{0}$ is assumed to be proportional to the amplitude $z_{0}^{\mathrm{i}}$ of the incident wave at the point of diffraction $\mathbf{x}_{0}(\eta)$. Then $\delta_{0}=D z_{0}^{\mathrm{i}}\left[\mathbf{x}_{0}(\eta)\right]$ and

$$
\hat{z}_{0}(\sigma)=D z_{0}^{i}\left|\sigma\left(1+\frac{\sigma}{\rho}\right)\right|^{-\frac{1}{2}} .
$$

The factor of proportionality $D$ is called a diffraction coefficient. In Keller's theory it is determined by comparison with the solution of the problem of diffraction by a half-plane; the motivation is that diffraction is a local phenomenon and locally the screen can be approximated by a half-plane. In the next section we discuss the solution of the half-plane problem and derive the diffraction coefficient. The value of $D$ is given by (3.16). It depends on the angles $\beta, \varphi$, and $\varphi_{0}$. The angles $\varphi$ and $\varphi_{0}$ are illustrated in Fig. 1.

In closing this section we state and prove two lemmas which will be useful in Sec. 4 .

Lemma 1: Let $s^{\mathrm{i}, \mathrm{r}}$ be the phase function of the incident (reflected) wave and $\hat{s}$ the phase function of the diffracted wave. Then

$$
s^{i, r}(\mathbf{x}) \leq \hat{s}(\mathbf{x}),
$$

and $s^{\mathrm{i}, \mathrm{r}}(\mathbf{x})=\hat{s}(\mathbf{x})$ if and only if $\mathbf{x}$ is a point on the shadow boundary of the incident (reflected) wave.

Proof: Let $s_{0}$ be the common value of $s^{\mathrm{i}}, s^{\mathrm{r}}$, and $\hat{s}$ at a point $\mathbf{Q}$ on the edge. Let $\mathbf{P}$ be any point on a diffracted ray emanating from $\mathbf{Q}$ in the direction of the unit vector $\mathbf{U}$; and let $\sigma_{\mathbf{0}}$ be the distance from $\mathbf{Q}$ to $\mathbf{P}$. Since $\left|\boldsymbol{\nabla} \boldsymbol{s}^{\mathrm{i}, \mathrm{r}}\right|=1$,

$$
s^{\mathrm{i}, \mathrm{r}}(\mathbf{P})=s_{0}+\int_{\mathbf{0}}^{\mathbf{P}} \nabla s^{\mathrm{i}, \mathrm{r}} \cdot \mathbf{U} d \sigma \leq s_{0}+\sigma_{0}=\hat{s}(\mathbf{P}) .
$$

Equality holds in (2.37) if and only if $\nabla s^{\text {i.r }} \equiv \mathbf{U}$, i.e., if and only if the diffracted ray coincides with an incident (reflected) ray. But this occurs if and only if

FIG. 1. Angles at the edge of a screen. The unit vectors $t_{2}$ and $t_{3}$ are orthogonal to the unit vector $\mathbf{t}_{1}$, which is tangent to the edge. $t_{2}$ lies in the tangent plane and points away from the screen. $t_{3}=t_{1} \times t_{2}$ is orthogonal to the tangent plane and points toward the illuminated side. Projections of the incident ray and the diffracted ray into the plane orthogonal to the edge are shown. The directions of the projections are determined by the angles $\varphi_{0}\left(0 \leq \varphi_{0} \leq \pi\right)$ and $\varphi(-\pi<\varphi \leq \pi)$. For purposes of Sec. 3 the $y$ and $z$ axes are shown. For purposes of Secs. 3 and 5 the screen coincides with the tangent plane.

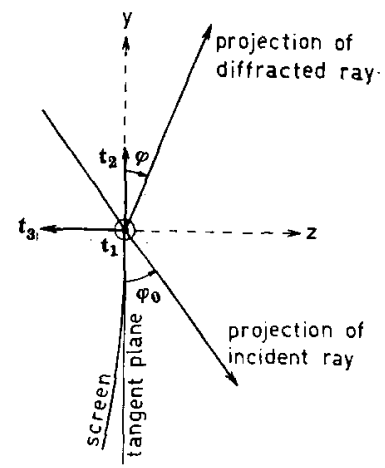


$\mathbf{P}$ is on the shadow boundary of the incident (reflected) wave.

Lemma 2: (See Fig. 2.) Let $\mathrm{t}$ be a unit vector tangent to the edge at $\mathbf{Q}$, and $\mathbf{U}_{1}$ a unit vector in the direction of the incident (reflected) ray at $Q$. Let $\mathbf{U}_{3}$ be a unit vector in the plane $T$ spanned by $t$ and $U_{1} \cdot U_{3}$ is chosen so that it is perpendicular to $U_{1}$ and

$$
\mathbf{t}=\cos \beta \mathbf{U}_{\mathbf{1}}+\sin \beta \mathbf{U}_{\mathbf{3}} .
$$

Let $\rho_{0}$ be the radius of curvature of the normal section of the incident (reflected) wavefront at $\mathbf{Q}$ in the direction of $\mathbf{U}_{3}$. Then for the diffracted ray emanating from $\mathbf{Q}$ which lies on the shadow boundary of the incident (reflected) wave, the quantity $\rho$ defined by $(2.31)$ has the value

$$
\rho=\rho_{0} \text {. }
$$

Proof: Let $S$ be the shadow-boundary surface of the incident (reflected) wave, $W(\mathbf{x})$ the incident (reflected) wavefront and $\hat{W}(\mathbf{x})$ the diffracted wavefront that passes through the point $\mathbf{x}$. On $S$, the incident (reflected) and diffracted rays coincide. (See Fig. 2.) Let $\mathbf{Q}$ denote a point on the edge $\mathbf{x}=\mathbf{x}_{0}(\eta)$ and $\mathbf{P}$ a point on the ray through $\mathbf{Q}$ at a distance $\sigma$ from $\mathbf{Q}$. From (2.9) and (2.30), we see that the principal radii at $\mathbf{P}$ of $W(\mathbf{P})$ are $\sigma$ and $\rho+\sigma$. The cone of diffracted rays emanating from $\mathbf{Q}$ intersects $\hat{W}(\mathbf{P})$ in a circle. The axis of the cone has the direction of the vector $t$. From the "formula of Rodrigues" it can easily be shown that the circle is a line of curvature on $\hat{W}(\mathbf{P})$ corresponding to the principal radius of curvature $\sigma$. Therefore one of the principal directions at $\mathbf{P}$ is tangent to the circle, hence perpendicular to $t$. Thus it is perpendicular to the plane $T$ spanned by $t$ and $\mathbf{U}_{1}$. The other principal direction corresponding to the principal radius of curvature $\rho+\sigma$ is given by the vector $\mathbf{U}_{3}$ which lies in the plane $T$ and is perpendicular to $\mathbf{U}_{\mathbf{1}}$. Since $\hat{W}(\mathbf{Q})$ and $\hat{W}(\mathbf{P})$ are parallel surfaces, their principal directions are the same. If we take $\sigma=0$, we see that the principal radius of curvature of $\hat{W}(\mathbf{Q})$ in the direction $\mathbf{U}_{3}$ is $\rho$.

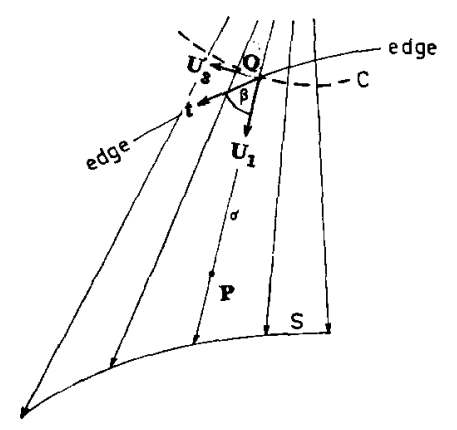

Fic. 2 The shadow boundary surface $S$ (proof of Lemma 2).
Let $C$ be the curve of intersection of $S$ and $W(\mathbf{Q})$. According to Lemma $1, \hat{s}=s$ on $S$; hence $C$ also lies on $\hat{W}(\mathbf{Q})$. The rays of $S$ are orthogonal to both $W(\mathbf{Q})$ and $\hat{W}(\mathbf{Q})$ along $C$; hence $W(\mathbf{Q})$ and $\hat{W}(\mathbf{Q})$ are tangent along $C$. From this it can easily be shown that the radii of curvature of the normal section of $W(\mathbf{Q})$ and $\hat{W}(\mathbf{Q})$ at any point of $C$ in the direction of the tangent to $C$ are equal. But at $\mathbf{Q}$ the tangent to $C$ has the direction $\mathbf{U}_{3}$. Hence $\rho_{0}=\rho$.

\section{SOMMERFELD'S SOLUTION OF THE PROBLEM OF DIFFRACTION BY A HALF-PLANE}

In this section we shall express Sommerfeld's wellknown solution of the half-plane diffraction problem in a new form. This expression partially motivated our work in this paper. From Sommerfeld's solution we shall also derive Keller's diffraction coefficient.

Let $x, y$, and $z$ be rectangular coordinates and let a half-plane be given by $z=0, y \leq 0$, as in Fig. 1 . We introduce polar coordinates $\rho, \varphi(0 \leq \rho ;-\pi \leq$ $\varphi \leq \pi)$ defined by the equations $y=\rho \cos \varphi, z=$ $\rho \sin \varphi ;$ and an incident plane wave $u_{0}^{\mathrm{i}}=\exp \left(i k s^{i}\right)$, where

$$
\begin{aligned}
s^{\mathrm{i}} & =x \cos \beta-y \sin \beta \cos \varphi_{0}+z \sin \beta \sin \varphi_{0} \\
& =x \cos \beta-\rho \sin \beta \cos \left(\varphi+\varphi_{0}\right) .
\end{aligned}
$$

For $\beta=\pi / 2$, the solution of the diffraction problem with boundary conditions (2.16) was first derived by Sommerfeld. A simple derivation appears in Ref. $10 .{ }^{11}$ For arbitrary $\beta$ the transformation

$$
u=\exp (i k x \cos \beta) u^{\prime}(y, z)
$$

reduces the general problem to the special one $(\beta=\pi / 2)$, with $k$ replaced by $k \sin \beta$. Thus it is not difficult to obtain the solution of the diffraction problem for the incident plane wave given above. We find that the total field is given by

$$
\begin{aligned}
u= & \exp \left\{i k\left[-\rho \sin \beta \cos \left(\varphi+\varphi_{0}\right)+x \cos \beta\right]\right\} \\
& \times h\left[(2 k \rho \sin \beta)^{\frac{1}{2}} \cos \frac{1}{2}\left(\varphi+\varphi_{0}\right)\right] \\
& \mp \exp \left\{i k\left[-\rho \sin \beta \cos \left(\varphi-\varphi_{0}\right)+x \cos \beta\right]\right\} \\
& \times h\left[-(2 k \rho \sin \beta)^{\frac{1}{2}} \cos \frac{1}{2}\left(\varphi-\varphi_{0}\right)\right],
\end{aligned}
$$

where

$$
h(x)=\pi^{-\frac{1}{2}} e^{-i \pi / 4} \int_{-\infty}^{x} e^{i t^{2}} d t .
$$


The above result can be greatly simplified by introducing the reflected and diffracted phase functions $s^{r}$ and $\hat{s}$ discussed in Sec. 2 . It is easily seen that

$$
\begin{aligned}
s^{r} & =x \cos \beta-y \sin \beta \cos \varphi_{0}-z \sin \beta \sin \varphi_{0} \\
& =x \cos \beta-\rho \sin \beta \cos \left(\varphi-\varphi_{0}\right) .
\end{aligned}
$$

The diffracted rays may be expressed in the form

$$
\begin{aligned}
\mathbf{x}=(x, y, z) & =(\eta, 0,0) \\
+ & \sigma(\cos \beta, \sin \beta \cos \varphi, \sin \beta \sin \varphi)
\end{aligned}
$$

and on each ray

$$
\hat{s}=s^{\mathrm{i}}(\eta, 0,0)+\sigma=\eta \cos \beta+\sigma .
$$

Since $\eta=x-\sigma \cos \beta$ and $\rho=\sigma \sin \beta$, we see that

$$
\hat{s}=x \cos \beta+\rho \sin \beta .
$$

We note that

$$
\hat{s}-s^{\mathbf{i}}=2 \rho \sin \beta \cos ^{2} \frac{1}{2}\left(\varphi+\varphi_{0}\right)
$$

and

$$
\hat{s}-s^{r}=2 \rho \sin \beta \cos ^{2} \frac{1}{2}\left(\varphi-\varphi_{0}\right) \text {. }
$$

We set

$$
\begin{aligned}
& \epsilon^{\mathrm{i}}=\operatorname{sgn}\left[\cos \frac{1}{2}\left(\varphi+\varphi_{0}\right)\right], \\
& \epsilon^{\mathrm{r}}=-\operatorname{sgn}\left[\cos \frac{1}{2}\left(\varphi-\varphi_{0}\right)\right],
\end{aligned}
$$

and

$$
f(x)=e^{-i x^{2}} h(x)=\pi^{-\frac{1}{2}} e^{-i \pi / 4} e^{-i x^{2}} \int_{-\infty}^{x} e^{i t^{2}} d t .
$$

Then (3.2) can be written as

$$
u=u^{\mathrm{i}}+u^{\mathrm{r}},
$$

where

$$
u^{i, r}=e^{i k \hat{s}} f\left\{\epsilon^{i, r}\left[k\left(\hat{s}-s^{i, r}\right)\right]^{\frac{1}{2}}\right\} z_{0}^{i, r},
$$

and $z_{0}^{\mathrm{i}}$ and $z_{0}^{\mathrm{r}}$ are the geometrical incident and reflected amplitudes. Thus

$$
z_{0}^{\mathrm{i}}=1, \quad z_{0}^{\mathrm{r}}=\mp 1 .
$$

The geometric structure of the exact solution [(3.12) and (3.13)] becomes even more striking when we recognize that $\epsilon^{i, r}=+1$ in the region illuminated by the incident (reflected) field and $\epsilon^{\mathbf{i}, \mathrm{r}}=-1$ in the shadow region of the incident (reflected) field.

On the shadow boundary of the incident (reflected) field, $\hat{s}=s^{i, r}$ and the argument of the function $f$ in (3.13) is zero. For points not on a shadow boundary, and for $k \rightarrow \infty$, we may introduce the asymptotic expansion of $f$ given in Appendix A. Then we find that $u \sim \eta\left(\epsilon^{\mathrm{i}}\right) e^{i k s^{1}} z_{0}^{\mathrm{i}}+\eta\left(\epsilon^{\mathrm{r}}\right) e^{i k s^{\mathrm{r}}} z_{0}^{\mathrm{r}}+e^{i k \hat{s}}(k \sigma)^{-\frac{1}{2}} D+O\left(k^{-\frac{3}{2}}\right)$,

where $\eta(x)$ is the unit-step function (see Appendix A) and

$$
D=-\frac{e^{i \pi / 4}}{2(2 \pi)^{\frac{1}{2}} \sin \beta}\left[\sec \frac{1}{2}\left(\varphi+\varphi_{0}\right) \pm \sec \frac{1}{2}\left(\varphi-\varphi_{0}\right)\right] .
$$

The factor $\eta\left(\epsilon^{\mathrm{i}, \mathrm{r}}\right)$ is one in the illuminated region of the incident (reflected) field and zero in the shadow region. Thus the first two terms in (3.15) are just the geometrical-optics solution. If we remember that the angle $\beta$ is constant on the edge and the curvature $\kappa$ of the edge is zero, we see that (2.31) yields $\rho=\infty$ and (2.35) becomes

$$
\hat{z}_{0}=\sigma^{-\frac{1}{2}} D z_{0}^{\mathrm{i}}=\sigma^{-\frac{1}{2}} D .
$$

Thus we recognize that the third term in (3.15) is the leading term of Keller's diffracted wave (2.23) and $D$ is the diffraction coefficient. Now, however, (3.16) provides a formula ${ }^{12}$ for $D$. In Keller's theory it is assumed that $D$ is given by (3.16) for an arbitrary screen, with the angle $\beta, \varphi$, and $\varphi_{0}$ defined at each point on the edge as in Fig. 1.

\section{DIFFRACTION BY A SCREEN}

In this section we reconsider the diffraction problem discussed in Sec. 2: The wave $u_{0}^{\mathrm{i}}$ given by $(2.15)$ is incident on an arbitrary smooth screen with either boundary condition (2.16). The total field $u$ is a solution of the reduced wave equation (2.1) and the boundary condition; and the scattered field $u-u_{0}^{\mathfrak{i}}$ must be outgoing. In addition $u$ must satisfy an "edge condition," ${ }^{13}$ which we shall introduce shortly.

As we have seen in Sec. 2, Keller's approach to this problem is based on the ansatz (2.2). Motivated by the representation (3.12) of the exact solution of the Sommerfeld problem, we shall introduce a new ansatz for the general diffraction problem. We will find that, away from the shadow boundaries, our results will reduce to those of Sec. 2 , but we shall be able to obtain more than just the leading term of the diffracted wave. (In this section we obtain the leading term. In Sec. 5 we obtain the next term, and presumably the process can be continued.) Moreover, we shall not resort to the Sommerfeld solution for the determination of the diffraction coefficient. Instead, we shall find that the coefficient and its generalization for higher-order terms arise as a consequence of

\footnotetext{
${ }^{12}$ If we introduce Keller's angles, $\alpha=\pi / 2-\varphi_{0}, \theta=\pi / 2+\varphi$, we see that (3.16) agrees with the result given in Ref. 1 or Ref. 2. In these references the factor $k^{-\frac{1}{2}}$ is included in the diffraction coefficient. We prefer to define $D$ so that it is dimensionless.

13 It is well known that an edge condition is necessary for the solution to be unique. [See C. J. Bouwkamp, Rept. Progr. Phys. 17, 35 (1954).] The Sommerfeld solution in Sec. 3 satisfies this condition. The condition also enters indirectly into the method of Sec. 2 because Keller's diffraction coefficient is obtained from the Sommerfeld solution.
} 
imposing the edge condition on our asymptotic solution. We will also see that the asymptotic solution obtained in this section will be continuous at the shadow boundaries as well as at the edge. For this reason we call it "uniform."

Our asymptotic solution is based on the ansatz

$$
u=u^{\mathrm{i}}+u^{\mathrm{r}}
$$

where

$$
\begin{aligned}
u^{\mathrm{i}, \mathrm{r}} \sim e^{i k \hat{s}}\left(f\left\{\epsilon^{i, \mathrm{r}}\left[k\left(\hat{s}-s^{\mathrm{i}, \mathrm{r}}\right)\right]^{\frac{1}{2}}\right\} \sum_{m=0}^{\infty}(i k)^{-m} z_{m}^{\mathrm{i}, \mathrm{r}}\right. \\
\left.+k^{-\frac{1}{2}} \sum_{m=0}^{\infty}(i k)^{-m} w_{m}^{\mathrm{i}, \mathrm{r}}\right) .
\end{aligned}
$$

The function $f$ is given by (3.11). The second term in (4.2) is not present in (3.13), but such a term appears in the uniform asymptotic expansion of the solution of the problem of diffraction of a cylindrical wave by a half-plane. It is also suggested by the work of Wolfe. ${ }^{6}$ In (4.2) we take $\epsilon^{i, r}=1$ in the illuminated region of the incident (reflected) wave and $\epsilon^{i, r}=-1$ in the shadow region. For $\hat{s}-s^{\mathrm{i}, \mathrm{r}}>0$, we may introduce the asymptotic expansion of $f$ which is given in Appen$\operatorname{dix} \mathrm{A}$. Then we find that

$$
\begin{aligned}
u^{\mathrm{i}, \mathbf{r}} \sim \eta\left(\epsilon^{\mathrm{i}, \mathrm{r}}\right) e^{i k s^{1, \mathrm{r}}} \sum_{m=0}^{\infty}(i k)^{-m} z_{m}^{\mathrm{i}, \mathbf{r}} & \\
& +k^{-\frac{1}{2}} e^{i k \hat{s}} \sum_{m=0}^{\infty}(i k)^{-m} \hat{z}_{m}^{\mathrm{i}, \mathrm{r}},
\end{aligned}
$$

where $\hat{z}_{m}^{\mathrm{i}, \mathrm{r}}$ is determined by

$$
w_{m}^{\mathrm{i}, \mathbf{r}}=\hat{z}_{m}^{\mathrm{i}, \mathrm{r}}+\frac{\epsilon^{i, \mathbf{r}}}{2 \pi^{\frac{1}{2}}} e^{i \pi / 4} \sum_{n=0}^{m}\left(\frac{1}{2}\right)_{n} \frac{z_{m-n}^{\mathrm{i}, \mathbf{r}}}{\left(\hat{s}-s^{\mathrm{i}, \mathbf{r}}\right)^{n+\frac{1}{2}}} .
$$

In (4.3), $\eta\left(\epsilon^{\mathrm{i}, \mathrm{r}}\right)$ is one in the illuminated region of the incident (reflected) wave and is zero in the shadow. By inserting (4.2) into the reduced wave equation, one can obtain equations for the determination of $\hat{s}, s^{\mathrm{i}}, s^{\mathbf{r}}$, $z_{m}^{\mathrm{i}}, z_{m}^{\mathrm{r}}, w_{m}^{\mathrm{i}}, w_{m}^{\mathrm{r}}$. It is, however, much simpler to use (4.3) and then use (4.4) to obtain $w_{m}^{\mathrm{i}, \mathrm{r}}$.

It is clear from our work in Sec. 2 that (4.3) will satisfy the reduced wave equation, provided $s^{\mathrm{i}, \mathrm{r}}$ and $\hat{s}$ satisfy the eikonal equation; and both $z_{m}^{\mathrm{i}, \mathrm{r}}, s^{\mathrm{i}, \mathrm{r}}$ together, and $\hat{z}_{m}^{\mathrm{i}, \mathrm{r}}, \hat{s}$ together satisfy the transport equations (2.4).

We now impose the boundary conditions (2.16). If we insert $(4.1,4.3)$ into the first boundary condition $(u=0)$, we obtain $(2.18),(2.19)$, and the additional equations

$$
\hat{z}_{m}^{\mathrm{r}}=-\hat{z}_{m}^{\mathrm{i}} \quad \text { on } S, \quad m=0,1,2, \cdots .
$$

If we use the second boundary condition, we again obtain (2.18), (2.20), and the additional equations

$$
\begin{array}{r}
\frac{\partial \hat{s}}{\partial n}\left[\hat{z}_{m}^{\mathrm{i}}+\hat{z}_{m}^{\mathrm{r}}\right]+\frac{\partial \hat{z}_{m-1}^{\mathrm{i}}}{\partial n}+\frac{\partial \hat{z}_{m-1}^{\mathrm{r}}}{\partial n}=0 \quad \text { on } S, \\
m=0,1,2, \cdots .
\end{array}
$$

Since the functions $s^{\mathrm{r}}$ and $z_{m}^{\mathrm{r}}$ satisfy exactly the same equations as those of Sec. 2 , these functions are identical to those of Keller's theory and may be computed by the method of Sec. 2. The functions $s^{i}$ and $z_{m}^{1}$ are, of course, given. As in Sec. 2, we assume that the diffracted rays emanate from the edge and that $\hat{s}=s^{i}$ on the edge. It follows that $\hat{s}$ is identical to the diffracted-phase function of Sec. 2. Furthermore, since $\hat{z}_{m}^{i, r}$ and $\hat{s}$ together satisfy the transport equations, $\hat{z}_{m}^{i, r}$ is given along the diffracted rays by (2.33) with $\delta_{m}$ replaced by $\delta_{m}^{\mathrm{i}, \mathrm{r}}$. These coefficients will be determined shortly by the edge condition: $u$ must have a finite limit at the edge. ${ }^{14}$

Once all of the functions in (4.3) are determined, (4.2) follows from (4.4). Since $\hat{s}$ and $s^{\mathrm{i}, \mathrm{r}}$ have been identified as the phase functions of Sec. 2 , it follows from Lemma 1 that $\hat{s}-s^{i, r} \geq 0$ and $\hat{s}=s^{i, r}$ only on the shadow boundary. We take the radical in (4.2) to mean the nonnegative square root.

We may assume that the functions $s^{\mathrm{i}}$ and $z_{m}^{\mathrm{i}}$ are defined everywhere. However, the functions $s^{\mathrm{r}}$ and $z_{m}^{\mathrm{r}}$ are so far defined only in the illuminated region of the reflected wave. In order for (4.2) to be defined everywhere, we must continue the reflected wave into its shadow region, i.e., we must continue the functions $s^{\mathrm{r}}$ and $z_{m}^{\mathrm{r}}$. We require that these continuations be smooth (i.e., the functions must have sufficiently many derivatives). This smooth continuation can easily be constructed by continuing the screen smoothly past the edge and extending the reflected rays backward through the screen. Then $s^{\mathrm{r}}$ and $z_{m}^{\mathrm{r}}$ are defined along these extended rays by the formulas of Sec. 2. As a consequence they will satisfy the eikonal and transport equations in the shadow region. (If the screen has an analytic representation, we can use its analytic continuation.) It can be shown that, to any given order in $k^{-1}$, the asymptotic expansion (4.2) for $u^{\mathrm{r}}$ is independent of the continuation of $s^{\mathrm{r}}$ and $z_{m}^{\mathrm{r}}$, provided it is sufficiently smooth. ${ }^{15}$ In fact, if $k\left(\hat{s}-s^{r}\right) \gg 1$, it is clear from (4.3) that $u^{\mathrm{r}}$ does not depend on values of $s^{\mathrm{I}}$ and $z_{m}^{\mathrm{r}}$ in the shadow, for there $\eta\left(\epsilon^{\mathrm{T}}\right)=0$. For points so close to the shadow boundary that $k\left(\hat{s}-s^{\mathrm{r}}\right)$ is finite, it can be shown that the distance to the shadow boundary is of order $k^{-\frac{1}{2}}$. Then, if (4.2) is sufficiently smooth, its values in the shadow are given by a Taylor expansion in the distance, hence

\footnotetext{
14 This condition is sufficient to ensure uniqueness of the solution of the diffraction problem. See C. J. Bouwkamp, Rept. Progr. Phys. 17, 35 (1954); L. M. Levine, Commun. Pure Appl. Math. 17, 147 (1964).

${ }^{15}$ This assertion depends on the assumption that the smoothness of $s^{\mathbf{r}}$ and $z_{m}^{\mathbf{r}}$ implies the smoothness of $w_{m}^{\mathbf{r}}$ at the shadow boundary. We will prove (Lemma 3 ) that $w_{0}^{r}$ is continuous at the shadow boundary. Further smoothness properties have not yet been proved.
} 
an expansion in powers of $k^{-\frac{1}{t}}$. The coefficients of the expansion up to any order depend only on derivatives at the shadow boundary, therefore are independent of the continuation provided it is sufficiently smooth. Thus any two continuations differ by a term of arbitrarily high order in $k^{-\frac{1}{2}}$, provided they are sufficiently smooth.

Since $f(x)$ is regular at $x=0$ and the functions $z_{m}^{\mathrm{i}, \mathrm{r}}$ are regular at the edge, we see from (4.2) that the edge condition is satisfied provided the equivalent edge condition, $\left|\lim _{\sigma \rightarrow 0} w_{m}^{\mathrm{i}, \mathrm{r}}\right|<\infty, m=0,1,2, \cdots$, on each diffracted ray, is satisfied. For $m=0$ we see that

$$
w_{0}^{i, r}=\hat{z}_{0}^{i, r}+\frac{\epsilon^{i, r}}{2} \pi^{-\frac{1}{2}} e^{i \pi / 4} \frac{z_{0}^{i, r}}{\left(\hat{s}-s^{i, r}\right)^{\frac{1}{2}}} .
$$

In order to apply the edge condition we now expand the functions that appear in (4.6) for small $\sigma$. From (2.34)

$$
\varepsilon_{0}^{i, r}=\delta_{0}^{i, r} \sigma^{-\frac{1}{2}}+O\left(\sigma^{\frac{1}{2}}\right) .
$$

Since the functions $z_{0}^{i, r}$ are regular at the edge $\mathbf{x}=$ $\mathbf{x}_{0}(\eta)$

$$
z_{0}^{\mathrm{i}, \mathrm{r}}=z_{0}^{\mathrm{i}, \mathrm{r}}\left[\mathbf{x}_{0}(\eta)\right]+O(\sigma) .
$$

In order to expand $s^{i, r}$ near the edge, we introduce the unit vectors $\mathbf{t}_{1}=\mathbf{t}=\dot{\mathbf{x}}_{0}(\eta), \mathbf{t}_{2}$, and $\mathbf{t}_{3}=\mathbf{t}_{1} \times \mathbf{t}_{\mathbf{2}}$ as illustrated in Fig. 1. Since $\nabla s^{i}$ is a unit vector, we may set [at $\mathbf{x}=\mathbf{x}_{0}(\eta)$ ]

$$
\boldsymbol{\nabla} s^{\mathrm{i}}=\cos \beta \mathbf{t}_{1}-\sin \beta \cos \varphi_{0} \mathbf{t}_{2}-\sin \beta \sin \varphi_{0} \mathbf{t}_{3} .
$$

This equation defines the angles $\beta(0 \leq \beta \leq \pi)$ and $\varphi_{0}\left(0 \leq \varphi_{0} \leq \pi\right)$. From the law of reflection it follows that

$$
\begin{array}{r}
\boldsymbol{\nabla} s^{\mathbf{r}}=\cos \beta \mathbf{t}_{1}-\sin \beta \cos \varphi_{0} \mathbf{t}_{2} \\
+\sin \beta \sin \varphi_{0} \mathbf{t}_{3},
\end{array}
$$

and by the law of edge diffraction, the diffracted rays are given by

where

$$
\mathbf{x}=\mathbf{x}_{0}(\eta)+\sigma \mathbf{U}
$$

$$
\begin{array}{r}
\mathbf{U}=\cos \beta \mathbf{t}_{1}+\sin \beta \cos \varphi \mathbf{t}_{2}-\sin \beta \sin \varphi \mathbf{t}_{3} \\
(-\pi \leq \varphi \leq \pi) .
\end{array}
$$

On a diffracted ray,

$$
\begin{aligned}
s^{\mathrm{i}, \mathbf{r}} & =s^{\mathrm{i}, \mathrm{r}}\left[\mathbf{x}_{0}(\eta)\right]+\sigma \mathrm{U} \cdot \nabla s^{\mathrm{i}, \mathbf{r}}+O\left(\sigma^{2}\right) \\
& =s^{\mathrm{i}}\left[\mathbf{x}_{0}(\eta)\right]+\sigma\left[\cos ^{2} \beta-\sin ^{2} \beta \cos \left(\varphi \pm \varphi_{0}\right)\right]
\end{aligned}
$$$$
+O\left(\sigma^{2}\right) \text {. }
$$

Hence, since $\hat{s}=s^{\mathrm{i}}\left[\mathbf{x}_{0}(\eta)\right]+\sigma$,

$$
\begin{aligned}
\epsilon^{\mathrm{i}, \mathrm{r}}\left(\hat{s}-s^{\mathrm{i}, \mathrm{r}}\right)^{\frac{1}{2}} \\
\quad= \pm(2 \sigma)^{\frac{1}{2}} \sin \beta \cos \frac{1}{2}\left(\varphi \pm \varphi_{0}\right)+O\left(\sigma^{\frac{3}{2}}\right) .
\end{aligned}
$$

Here we have set

$$
\epsilon^{\mathrm{i} . \mathrm{r}}= \pm \operatorname{sgn}\left[\cos \frac{1}{2}\left(\varphi \pm \varphi_{0}\right)\right],
$$

and it can be easily verified that $\epsilon^{\mathrm{i}, \mathrm{r}}$ is indeed +1 in the illuminated region of the incident (reflected) wave and -1 in the shadow. By inserting (4.7), (4.8), and (4.14) into (4.6), we find that (for $m=0$ ) the edge condition is satisfied provided

$$
\delta_{0}^{i, r}=\mp \frac{e^{i \pi / 4}}{2(2 \pi)^{\frac{1}{2}}} \frac{z_{0}^{i, r}\left[x_{0}(\eta)\right]}{\sin \beta \cos \frac{1}{2}\left(\varphi \pm \varphi_{0}\right)} .
$$

We may now express $z_{0}^{\mathrm{r}}\left[\mathbf{x}_{0}(\eta)\right]$ in terms of $z_{0}^{\mathrm{i}}\left[x_{0}(\eta)\right]$ by using (2.19) or (2.21). (For $m=0$, the latter becomes simply $z_{0}^{r}=z_{0}^{i}$.) Then from (2.34) we have, for the boundary condition $u=0(\partial u / \partial n=0)$ on $S$,

$$
\hat{z}_{0}^{i}(\sigma)=-\frac{e^{i \pi / 4}}{2(2 \pi)^{\frac{1}{2}}} \frac{z_{0}^{\mathrm{i}}\left[\mathbf{x}_{0}(\eta)\right]}{\sin \beta \cos \frac{1}{2}\left(\varphi+\varphi_{0}\right)}\left|\sigma\left(1+\frac{\sigma}{\rho}\right)\right|^{-\frac{1}{2}}
$$

and

$$
\begin{gathered}
\hat{z}_{0}^{\mathrm{r}}(\sigma)=\frac{e^{i \pi / 4}}{2(2 \pi)^{\frac{1}{2}}} \frac{z_{0}^{\mathrm{r}}\left[\mathbf{x}_{0}(\eta)\right]}{\sin \beta \cos \frac{1}{2}\left(\varphi-\varphi_{0}\right)}\left|\sigma\left(1+\frac{\sigma}{\rho}\right)\right|^{-\frac{1}{2}} \\
z_{0}^{\mathrm{r}}\left[\mathbf{x}_{0}(\eta)\right]=\mp z_{0}^{\mathrm{i}}\left[\mathbf{x}_{0}(\eta)\right] .
\end{gathered}
$$

Thus we have found the terms of (4.3) and (4.2) for which $m=0$. The leading term of the nonuniform expansion $(4.1,4.3)$ is just the geometrical-optics (incident and reflected) field. The second term is given by

where

$$
\hat{u} \sim k^{-\frac{1}{2}} e^{i k \hat{z}} \hat{z}_{0},
$$

$$
\hat{z}_{0}=\hat{z}_{0}^{\mathrm{i}}+\hat{z}_{0}^{\mathrm{r}}=D z_{0}^{\mathrm{i}}\left[\mathbf{x}_{0}(\eta)\right]\left|\sigma\left(1+\frac{\sigma}{\rho}\right)\right|^{-\frac{1}{2}},
$$

and, for the boundary condition $u=0(\partial u / \partial n=0)$,

$D=-\frac{e^{i \pi / 4}}{2(2 \pi)^{\frac{1}{2}} \sin \beta}\left[\sec \frac{1}{2}\left(\varphi+\varphi_{0}\right) \pm \sec \frac{1}{2}\left(\varphi-\varphi_{0}\right)\right]$.

From (3.16) we see that $D$ is Keller's diffraction coefficient, and, by comparing (4.20) with (2.35) and (4.19) with (2.23), we see that away from the shadow boundary our result reduces to the solution obtained by the geometrical theory of diffraction.

The terms of the uniform expansion [(4.1) and (4.2)] obtained so far are given by (4.1) and

$$
u^{i, r} \sim e^{i k \hat{s}}\left(f\left\{\epsilon^{i, r}\left[k\left(\hat{s}-s^{i, r}\right)\right]^{\frac{1}{2}}\right\} z_{0}^{i, r}+k^{-\frac{1}{2}} w_{0}^{i, r}\right) .
$$

Here $w_{0}^{\mathrm{i}, \mathrm{r}}$ is given by (4.6), (4.17), and (4.18). Let us compare the present solution (4.22) with Keller's solution. By construction, the solution (4.22) is finite at the edge because we have satisfied the edge condition, whereas Keller's geometrical theory fails at the 
edge. However, at all other caustic points of the diffracted wave, as well as caustic points of the incident and reflected waves, both our solution and Keller's solution will fail. At the shadow boundary, the geometrical theory breaks down because the geometrical-optics field is discontinuous and the diffraction coefficient becomes infinite. We will now verify that the uniform solution (4.22) is continuous at the shadow boundary. Since $f(x)$ is regular at $x=0$ and $z_{0}^{\mathrm{i}, \mathrm{r}}$ is smooth, it is sufficient to examine only the second term of (4.22) which is given by (4.6). At the shadow boundary of the incident (reflected) wave, $\hat{s}-s^{\mathrm{i}, \mathrm{r}}=0$ and $\cos \frac{1}{2}\left(\varphi \pm \varphi_{0}\right)=0$; hence both terms in (4.6) become infinite and we must evaluate the limit of the sum carefully. This is done in Appendix $B$, where we prove the following:

Lemma 3: $w_{0}^{\mathrm{i}, \mathrm{r}}$ is continuous at the shadow boundary of the incident (reflected) wave.

It follows that the leading term [(4.1), (4.2)] of our solution [(4.1), (4.2)] is continuous everywhere except at caustics of the incident and reflected waves and any caustic of the diffracted wave other than the edge.

We have not yet examined Eqs. (4.5a) and (4.5b), which must be satisfied if (4.1) is to satisfy the boundary condition (2.16a) and (2.16b). We find in the next section that these equations are indeed satisfied if the screen is a portion of a plane, provided none of the diffracted rays strike another part of the edge. If that occurs, it is necessary to introduce additional terms into (4.1). Examples of such problems of multiple diffraction will be treated by our method in a forthcoming paper. They are treated by Keller's method in Refs. 1 and 2. If the screen is curved, still more terms must be introduced into (4.1) if the boundary condition is to be satisfied. These terms correspond to secondary reflected waves which are produced when reflected or diffracted rays strike the screen at an angle of incidence less than $\pi / 2$, and creeping waves ${ }^{7}$ which are excited when incident, reflected, or diffracted rays are tangent to a convex portion of the screen. The existence of such terms was suggested by earlier experience with asymptotic methods in other problems but has not yet been verified for our problem.

\section{DIFFRACTION BY A PLANE SCREEN}

In order to compute higher-order terms in our expansion (4.1) and (4.2) or (4.1) and (4.3), we may use (2.33) for $\hat{z}_{m}^{\mathrm{i}, \mathrm{r}}$. Thus to determine $\hat{z}_{1}^{\mathrm{i}, \mathrm{r}}$ it is necessary to find $\Delta \hat{z}_{0}^{\hat{i}, r}$. But $\hat{z}_{0}^{\hat{i}, r}$ is given by (4.17) and (4.18) as a function of $\sigma, \varphi, \eta$. Thus it is necessary to transform the Laplacian to these coordinates which are not orthogonal. In order to simplify this and other parts of our work, we specialize our problem and consider only plane screens in this section.

The screen lies in the plane $x_{3}=0$ and is bounded by a smooth curve $\mathbf{x}=\mathbf{x}_{0}(\eta)=\left(x_{0}^{1}, x_{0}^{2}, 0\right)$. $\eta$ denotes arclength on the edge; hence $t_{1}=\dot{\mathbf{x}}_{\mathbf{0}}$ is a unit tangent vector. A dot denotes differentiation with respect to $\eta$. We assume that the incident wave comes from the region $x_{3}<0$. Then the $x_{3}$ axis coincides with the $z$ axis of Fig. 1 and $t_{3}=(0,0,-1)$. The parameter $\eta$ is chosen in such a way that the vector $t_{2}=t_{3} \times t_{1}$ points away from the screen as in Fig. 1. If $\mathbf{n}$ denotes the unit normal to the edge, then $\mathbf{t}_{\mathbf{2}}= \pm \mathbf{n}$ and the upper (lower) sign holds when the screen is locally concave (convex). In either case the curvature $\kappa$ is given by

where

$$
\kappa=\mathbf{n} \cdot \dot{\mathbf{t}}_{1}=\mp \tilde{\kappa},
$$

$$
\tilde{\kappa}=\dot{x}_{0}^{1} \ddot{x}_{0}^{2}-\dot{x}_{0}^{2} \ddot{x}_{0}^{1}
$$

is the "signed curvature." Then

$$
\dot{\mathbf{t}}_{1}=\kappa \mathbf{n}=-\tilde{\kappa} \mathrm{t}_{2}, \quad \dot{\mathbf{t}}_{2}=\tilde{\kappa} \mathbf{t}_{1}, \quad \dot{\mathbf{t}}_{\mathbf{3}}=0 .
$$

From (4.11) and (4.12) the diffracted rays are given by

where

$$
\mathbf{x}=\mathbf{x}_{0}(\eta)+\sigma \mathbf{U}(\eta, \varphi),
$$

$$
\mathbf{U}=\cos \beta \mathbf{t}_{1}+\sin \beta \cos \varphi \mathbf{t}_{2}-\sin \beta \sin \varphi \mathbf{t}_{3},
$$

and $\beta=\beta(\eta)$. By comparing (5.5) with (2.29), we see that $\cos \alpha= \pm \cos \varphi$; hence (2.31) yields

$$
\rho=\sin \beta /(\tilde{\kappa} \cos \varphi-\dot{\beta}) .
$$

By using (5.3), (5.6), and standard formulas, ${ }^{16}$ the Laplacian can be computed in $\sigma, \varphi, \eta$ coordinates. This is done in Appendix C. We will soon make use of the final result of that appendix, which is an expansion of the Laplacian for small $\sigma$.

For the case of a plane screen considered here, we can give a simplified representation of the reflected wave. It is easy to show that now

$$
s^{\mathrm{I}}\left(x_{1}, x_{2}, x_{3}\right)=s^{\mathrm{i}}\left(x_{1}, x_{2},-x_{3}\right),
$$

and, for the two boundary conditions $u=0$ and $\partial u / \partial n=\partial u / \partial x_{3}=0$

$$
z_{m}^{\mathrm{r}}\left(x_{1}, x_{2}, x_{3}\right)=\mp z_{m}^{\mathrm{i}}\left(x_{1}, x_{2},-x_{3}\right) .
$$

To verify (5.7) and (5.8) we note that $s^{\mathrm{r}}$ satisfies the eikonal equation (2.3) because $s^{i}$ does. Furthermore, $s^{\mathrm{r}}$ and $z_{m}^{\mathrm{r}}$ satisfy the system of transport equations (2.4) because $s^{\mathrm{i}}$ and $z_{m}^{\mathrm{i}}$ satisfy the same system.

${ }^{16}$ J. A. Stratton, Electromagnetic Theory (McGraw-Hill Book Co., New York, 1964). 
Finally, for the boundary condition $u=0,(2.19)$ is clearly satisfied, and for the boundary condition $\partial u / \partial n=0,(2.21)$ is satisfied because $\left(\partial / \partial x_{3}\right) z_{m}^{\mathrm{r}}+$ $\left(\partial / \partial x_{\mathfrak{a}}\right) z_{m}^{1}=0$ on the screen. For both boundary conditions $(2.18)$ is satisfied.

The functions $\hat{z}_{1}^{1, \mathrm{r}}$ are given by (2.33) for $m=1$ once the coefficients $\delta_{1}^{\mathrm{i}, \mathrm{r}}$ are determined. As in the evaluation of $\delta_{0}^{\mathrm{i}, \mathrm{r}}$, the values of $\delta_{1}^{\mathrm{i}, \mathrm{r}}$ are uniquely determined by the edge condition. To apply this condition it is necessary to compute [cf. (4.4)]

$$
w_{1}^{\mathbf{i}, \mathbf{r}}=\hat{z}_{1}^{i, \mathrm{r}}+\frac{\epsilon^{\mathrm{i}, \mathbf{r}}}{2 \pi^{\frac{1}{2}}} e^{i \pi / 4}\left[\frac{z_{1}^{\mathrm{i}, \mathrm{r}}}{\left(\hat{s}-s^{\mathrm{i}, \mathrm{r}}\right)^{\frac{1}{2}}}+\frac{1}{2} \frac{z_{0}^{\mathrm{i}, \mathrm{r}}}{2\left(\hat{s}-s^{\mathrm{i}, \mathrm{r}}\right)^{\frac{3}{2}}}\right]
$$

for small values of $\sigma$. Then the edge condition, $\left|\lim w_{1}^{\mathrm{i}, \mathrm{r}}\right|<\infty$, will determine the value of $\delta_{1}^{\mathrm{i}, \mathrm{r}}$. The first step is to find $\hat{z}_{1}^{i, r}$ for small $\sigma$ and this requires the determination of $\Delta \hat{z}_{0}^{\mathrm{i}, \mathrm{r}}$ for small $\sigma$. Therefore we apply (C16) to the first two terms of the expansion of (4.17) and (4.18) for small values of $\sigma$. The computation, although somewhat long, is straightforward and the surprisingly simple result is

$$
\begin{aligned}
\Delta \hat{z}_{0}^{i, \mathbf{r}}=\frac{\mp 1}{4(2 \pi)^{\frac{1}{2}}} e^{i \pi / 4} \frac{z_{0}^{\mathrm{i}, \mathrm{r}}\left[\mathbf{x}_{0}(\eta)\right]}{\sin ^{3} \beta \cos ^{3} \frac{1}{2}\left(\varphi \pm \varphi_{0}\right)} \\
\times\left[\sigma^{-\frac{5}{2}}-\frac{1}{2 \rho} \sigma^{-\frac{3}{2}}+O\left(\sigma^{-\frac{1}{2}}\right)\right] .
\end{aligned}
$$

The functions $\hat{z}_{1}^{\mathrm{i}, \mathrm{r}}$ are now given by [cf. (2.33)]

$$
\hat{z}_{\mathbf{1}}^{\mathbf{i}, \mathbf{r}}=\left[\sigma\left(1+\frac{\sigma}{\rho}\right)\right]^{-\frac{1}{2}}\left\{\delta_{1}^{\mathbf{i}, \mathrm{r}}-\frac{1}{2} f_{0}^{\sigma} \mathrm{g}^{\mathbf{i}, \mathbf{r}}(t) d t\right\},
$$

where, from (5.10),

$$
\begin{aligned}
g^{\mathrm{i}, \mathrm{r}}(t)= & {\left[t\left(1+\frac{t}{\rho}\right)\right]^{\frac{1}{2}} \Delta \hat{z}_{0}^{\mathrm{i}, \mathrm{r}}(t) } \\
= & \mp \frac{1}{4(2 \pi)^{\frac{1}{2}}} e^{i \pi / 4} \frac{z_{0}^{\mathrm{i}, \mathrm{r}}\left[\mathbf{x}_{0}(\eta)\right]}{\sin ^{3} \beta \cos ^{3} \frac{1}{2}\left(\varphi \pm \varphi_{0}\right)} \\
& \times\left[t^{-2}+O(1)\right] .
\end{aligned}
$$

The finite part integral in (5.11) is now easily evaluated. (We need only ignore the infinite contribution from the lower limit of integration.) Thus we obtain

$$
\begin{array}{r}
\hat{z}_{1}^{i, \mathbf{r}}=\delta_{1}^{i, \mathbf{r}} \sigma^{-\frac{1}{2}} \mp \frac{1}{8(2 \pi)^{\frac{1}{2}}} e^{i \pi / 4} \frac{z_{0}^{i, \mathbf{r}}\left[\mathbf{x}_{0}(\eta)\right]}{\sin ^{3} \beta \cos ^{3} \frac{1}{2}\left(\varphi \pm \varphi_{0}\right)} \\
\times\left[\sigma^{-\frac{3}{2}}-\frac{\sigma^{-\frac{1}{2}}}{2 \rho}\right]+O\left(\sigma^{\frac{1}{2}}\right) .
\end{array}
$$

It is interesting to note that the term of order $t^{-1}$ in (5.12) is missing. Such a term would have led to a term involving $\log \sigma$ in (5.13) and then the edge condition could not have been satisfied.
In order to compute the remaining terms in (5.9) for small $\sigma$, we note that $z_{0}^{\mathrm{i}, \mathrm{r}}$ and $z_{1}^{\mathrm{i}, \mathrm{r}}$ are regular in a neighborhood of the edge; hence

and

$$
z_{1}^{\mathrm{i}, \mathrm{r}}=z_{1}^{\mathrm{i}, \mathrm{r}}\left[\mathbf{x}_{0}(\eta)\right]+O(\sigma)
$$

where

$$
z_{0}^{\mathrm{i}, \mathbf{r}}=z_{0}^{\mathrm{i}, \mathrm{r}}\left[\mathbf{x}_{0}(\eta)\right]+a^{\mathrm{i}, \mathrm{r}} \sigma+O\left(\sigma^{2}\right),
$$

$$
a^{\mathbf{i}, \mathbf{r}}=\mathbf{U}(\eta, \varphi) \cdot \nabla z_{0}^{i, \mathbf{r}}\left[\mathbf{x}_{0}(\eta)\right] .
$$

Then, by extending (4.13) to one more term, we obtain

$$
\begin{aligned}
s^{\mathrm{i}, \mathrm{r}} & =s^{\mathrm{i}}\left[\mathbf{x}_{0}(\eta)\right] \\
+ & \sigma\left[\cos ^{2} \beta-\sin ^{2} \beta \cos \left(\varphi \pm \varphi_{0}\right)\right]+b^{\mathrm{i}, \mathrm{r}} \sigma^{2}+O\left(\sigma^{3}\right),
\end{aligned}
$$

where

$$
2 b^{\mathrm{i}, \mathrm{r}}=(\mathbf{U} \cdot \boldsymbol{\nabla})(\mathbf{U} \cdot \nabla) s^{\mathrm{i}, \mathrm{r}}=(\mathbf{U} \cdot \boldsymbol{\nabla})^{2} s^{\mathrm{i}, \mathbf{r}} .
$$

Since $s=s^{\mathrm{i}}\left[\mathbf{x}_{0}(\eta)\right]+\sigma$, we find that

$\hat{s}-s^{\mathrm{i}, \mathrm{r}}=2 \sigma \sin ^{2} \beta \cos ^{2} \frac{1}{2}\left(\varphi \pm \varphi_{0}\right)$

$$
-b^{\mathrm{i}, \mathrm{r}} \sigma^{2}+O\left(\sigma^{3}\right) .
$$

It is now an easy matter to compute $\left(\hat{s}-s^{\mathrm{i}, \mathrm{r}}\right)^{-\frac{1}{2}}$ and $\left(\hat{s}-s^{\mathrm{i}, \mathrm{r}}\right)^{-\frac{3}{2}}$. Then we may evaluate (5.9) for small $\sigma$. In so doing we must use (4.15). It is clear that the result will be of the form

$$
w_{1}^{\mathrm{i}, \mathrm{r}}=p_{1}^{\mathrm{i}, \mathrm{r}} \sigma^{-\frac{3}{2}}+p_{2}^{\mathrm{i}, \mathrm{r}} \sigma^{-\frac{1}{2}}+O\left(\sigma^{\frac{1}{2}}\right),
$$

but it is remarkable that the calculation yields

$$
p_{1}^{i, r}=0 .
$$

Then the edge condition will be satisfied if and only if $p_{2}^{\mathrm{i}, \mathrm{r}}=0$, and this condition uniquely determines $\delta_{\mathbf{1}}^{\mathrm{i}, \mathrm{r}}$. The result is

$$
\begin{aligned}
\delta_{1}^{\mathrm{i}, \mathrm{r}}= & \frac{\mp e^{i \pi / 4}}{2(2 \pi)^{\frac{1}{2}} \sin \beta}\left\{\frac{z_{1}^{\mathrm{i}, \mathrm{r}}\left(\mathbf{x}_{0}\right)}{\cos \frac{1}{2}\left(\varphi \pm \varphi_{0}\right)}\right. \\
& +\frac{1}{4 \sin ^{2} \beta \cos ^{3} \frac{1}{2}\left(\varphi \pm \varphi_{0}\right)}\left[a^{\mathrm{i}, \mathrm{r}}+\frac{1}{2 \rho} z_{0}^{\mathrm{i}, \mathrm{r}}\left(\mathbf{x}_{0}\right)\right] \\
& \left.+\frac{3 b^{i, \mathrm{r}} z_{0}^{\mathrm{i}, \mathrm{r}}\left(\mathbf{x}_{0}\right)}{16 \sin ^{4} \beta \cos ^{5} \frac{1}{2}\left(\varphi \pm \varphi_{0}\right)}\right\}
\end{aligned}
$$

With this value of $\delta_{1}^{\mathrm{i}, \mathrm{r}}, \hat{z}_{1}^{\mathrm{i}, \mathrm{r}}$ is given by (2.33) for $m=1$.

We note from $(5.20)$ that the expansion for small $\sigma$ of $w_{1}^{\mathrm{i}, \mathrm{r}}$ has two terms which become infinite at $\sigma=0$. One of them automatically vanishes, and the vanishing of the other term, which is required by the edge condition, uniquely determines $\delta_{1}^{\mathrm{i}, \mathrm{r}}$. In general it can be seen that the expansion of $w_{m}^{\mathrm{i}, \mathrm{r}}$ for small $\sigma$ will be of the form

$$
\begin{aligned}
w_{m}^{\mathrm{i}, \mathrm{r}}=p_{1}^{\mathrm{i}, \mathrm{r}} \sigma^{-m-\frac{1}{2}} & +p_{2}^{\mathrm{i}, \mathrm{r}} \sigma^{-m+\frac{1}{2}} \\
& +\cdots+p_{m+1}^{\mathrm{i}, \mathrm{r}} \sigma^{-\frac{1}{2}}+O\left(\sigma^{\frac{1}{2}}\right) .
\end{aligned}
$$


We conjecture that the first $m$ terms will vanish automatically, i.e., $p_{j}^{\mathrm{i}, \mathrm{r}}=0$ for $j=1,2, \cdots, m$. Then the vanishing of the remaining term, i.e., the requirement $p_{m+1}^{\mathrm{i}, \mathrm{r}}=0$, will uniquely determine $\delta_{m}^{\mathrm{i} . \mathrm{r}}$. But this conjecture has not yet been proved.

While in the realm of conjecture, we should also consider the behavior of our asymptotic solution $(4.1,4.2)$ in the neighborhood of the shadow boundaries. At the end of Sec. 4 we proved that the leading term $(4.1,4.22)$ is continuous at the shadow boundaries as well as at the edge. We also conjecture that to every order $(4.1,4.2)$ is not only continuous but smooth at the shadow boundaries. This conjecture also has not yet been verified. (Note added in proof: As mentioned in Sec. 1, both conjectures have recently been proven.) (At the edge, derivatives of the asymptotic solution may become singular, but the exact solution has the same property.)

Our nonuniform expansion [(4.1) and (4.3)] may be written in the form

where

$$
u=\eta\left(\epsilon^{\mathrm{i}}\right) u_{0}^{\mathrm{i}}+\eta\left(\epsilon^{\mathrm{r}}\right) u_{0}^{\mathrm{r}}+\hat{u},
$$

$$
\begin{gathered}
u_{0}^{\mathrm{i}, \mathrm{r}} \sim e^{i k s^{1, \mathrm{r}}} \sum_{m=0}^{\infty}(i k)^{-m} z_{m}^{\mathrm{i}, \mathrm{r}}, \\
\hat{u} \sim k^{-\frac{1}{2}} e^{i k \delta} \sum_{m=0}^{\infty}(i k)^{-m} \hat{z}_{m},
\end{gathered}
$$

and

$$
\hat{z}_{m}=\hat{z}_{m}^{\mathrm{i}}+\hat{z}_{m}^{\mathrm{r}} \text {. }
$$

By comparing (5.23)-(5.25) with the results of Sec. 2, we find that they are identical. In Sec. 4 we showed that the leading. term of the diffracted wave (5.25) agrees exactly with Keller's formula. The geometrical theory of diffraction (Sec. 2) is not capable of determining higher-order terms in (5.25), but our theory yields these terms as well. ${ }^{17}$

Since $\hat{z}_{m}^{\mathrm{i}}$ and $\hat{z}_{m}^{\mathrm{r}}$ both satisfy (2.33) with $\delta_{m}$ replaced by $\delta_{m}^{\mathrm{i}}$ and $\delta_{m}^{\mathrm{r}}$, it is clear from (5.26) that $\hat{z}_{m}$ satisfies (2.33) with

$$
\delta_{m}=\delta_{m}^{\mathrm{i}}+\delta_{m}^{\mathrm{r}} .
$$

Since we have determined $\delta_{1}^{\mathrm{i}}$ and $\delta_{1}^{\mathrm{r}}$, we can give the value of $\delta_{1}$. First, however, we simplify the terms $a^{\mathrm{i}, \mathrm{r}}$ and $b^{\mathrm{i}, \mathrm{r}}$ that appear in (5.22). We introduce the tangential- and normal-gradient operators defined by

$$
\begin{gathered}
\boldsymbol{\nabla}_{n}=\mathbf{N N} \cdot \boldsymbol{\nabla}, \quad \nabla_{t}=\boldsymbol{\nabla}-\boldsymbol{\nabla}_{n}, \\
\mathbf{N}=(0,0,1)=-\mathbf{t}_{\mathbf{3}} .
\end{gathered}
$$

Then (5.7) and (5.8) yield

$$
\begin{array}{rlrl}
\boldsymbol{\nabla}_{n} s^{\mathrm{r}} & =-\boldsymbol{\nabla}_{n} s^{\mathrm{i}}, & \boldsymbol{\nabla}_{t} s^{\mathrm{r}}=\boldsymbol{\nabla}_{t} s^{\mathrm{i}}, \\
\boldsymbol{\nabla}_{n} z_{m}^{\mathrm{r}}= \pm \boldsymbol{\nabla}_{n} z_{m}^{\mathrm{i}}, & \boldsymbol{\nabla}_{t} z_{m}^{\mathbf{r}}=\mp \nabla_{t} z_{m}^{\mathrm{i}},
\end{array}
$$

${ }^{17}$ The determination of the terms $z_{m}$ for $m=2,3, \cdots$ depends on the validity of our first conjecture. and from (5.5), (5.16), and (5.18) we obtain

$$
a^{i}=\mathbf{U} \cdot \nabla z_{0}^{i}, \quad a^{\mathbf{r}}=\mp \mathbf{U} \cdot \nabla z_{0}^{i} \pm 2 \sin \beta \sin \varphi \frac{\partial z_{0}^{\mathbf{i}}}{\partial n},
$$

and

$$
\begin{aligned}
& b^{\mathbf{i}}=\frac{1}{2}(\mathbf{U} \cdot \nabla)^{2} s^{\mathrm{i}} \\
& b^{\mathbf{r}}=\frac{1}{2}(\mathbf{U} \cdot \boldsymbol{\nabla})^{2} s^{i}-2\left(\mathbf{U} \cdot \nabla_{t}\right)\left(\mathbf{U} \cdot \nabla_{n}\right) s^{\mathbf{i}} .
\end{aligned}
$$

It now follows from (5.22) and (5.27) that

$$
\begin{aligned}
& \delta_{1}= \frac{-e^{i \pi / 4}}{2(2 \pi)^{\frac{1}{2}} \sin \beta} \\
& \quad \times\left\{\left[\sec \frac{1}{2}\left(\varphi+\varphi_{0}\right) \pm \sec \frac{1}{2}\left(\varphi-\varphi_{0}\right)\right] z_{1}^{\mathrm{i}}\left(\mathbf{x}_{0}\right)\right. \\
&+ \frac{1}{4} \csc ^{2} \beta\left[\sec ^{3} \frac{1}{2}\left(\varphi+\varphi_{0}\right) \pm \sec ^{3} \frac{1}{2}\left(\varphi-\varphi_{0}\right)\right] \\
& \times\left[\mathbf{U} \cdot \nabla z_{0}^{\mathrm{i}}\left(\mathbf{x}_{0}\right)+\frac{1}{2 \rho} z_{0}^{\mathrm{i}}\left(\mathbf{x}_{0}\right)\right]
\end{aligned}
$$$$
\times\left[\mathbf{U} \cdot \nabla z_{0}^{\mathrm{i}}\left(\mathbf{x}_{0}\right)+\frac{1}{2 \rho} z_{0}^{\mathrm{i}}\left(\mathbf{x}_{0}\right)\right]
$$$$
\times\left[(\mathbf{U} \cdot \boldsymbol{\nabla})^{2} s^{\mathrm{i}}\right] z_{0}^{\mathrm{i}}\left(\mathbf{x}_{0}\right)
$$

From (4.21) we see that (5.32) can be written in the form

$$
\delta_{1}=D z_{1}^{i}\left(\mathbf{x}_{0}\right)+D_{1} z_{0}^{\mathbf{i}}\left(\mathbf{x}_{0}\right)
$$

where $D$ is the "zero-order diffraction coefficient" (Keller's diffraction coefficient) and the "first-order diffraction coefficient" $D_{1}$ is a linear differential operator defined by (5.32).

It may happen that the incident rays are tangent to the screen. In this case of "grazing incidence," our results have some special features of interest. There are two cases to consider, depending on whether $\varphi_{0}=0$ or $\varphi_{0}=\pi$. (See Fig. 1.) For $\varphi_{0}=\pi$, the diffraction problem is pathological. This case will be treated in a sequel to the present paper.

If $\varphi_{0}=0$, the whole region is in the shadow of the reflected wave and illuminated by the incident wave, i.e., $\epsilon^{\mathrm{i}} \equiv 1$ and $\epsilon^{\mathrm{r}} \equiv-1$, and the shadow boundary coincides with the screen. As pointed out in Sec. 4, our results are independent of the values of the functions $z_{m}^{\mathrm{r}}$ and $s^{\mathrm{r}}$, provided those functions are sufficiently smooth. Therefore we may continue to define $z_{m}^{\mathrm{r}}$ and $s^{\mathrm{r}}$ by (5.7) and (5.8). For the boundary condition $\partial u / \partial n=0$, we see from (4.21) that $D=0$ and from (4.20) that $\hat{z}_{0} \equiv 0$. In this case, the leading term of the diffracted field (5.25) is given by

$$
\hat{u} \sim k^{-\frac{1}{2}} e^{i k \hat{s}}(i k)^{-1} \hat{z}_{1},
$$


and it is important to compute $\hat{z}_{1}$. This can easily be done now because $\nabla_{n} s^{\mathrm{i}} \equiv 0$ on $S$, and therefore (5.32) becomes

$$
\delta_{1}=\frac{-e^{i \pi / 4}}{4(2 \pi)^{\frac{1}{2}} \sin ^{2} \beta} \sin \varphi \sec ^{3} \frac{\varphi}{2} \frac{\partial z_{0}^{\mathrm{i}}}{\partial n}\left(\mathbf{x}_{0}\right) .
$$

Furthermore, since $\hat{z}_{0} \equiv 0$, the integral term in (2.33) is absent for $m=1$. It follows that

$$
\hat{u} \sim k^{-\frac{3}{2}} e^{i k \hat{s}} D^{\prime} \frac{\partial z_{0}^{1}}{\partial n}\left(\mathbf{x}_{0}\right)\left|\sigma\left(1+\frac{\sigma}{\rho}\right)\right|^{-\frac{1}{2}},
$$

where $D^{\prime}$ is a special diffraction coefficient given by

$$
D^{\prime}=-\frac{e^{-i \pi / 4}}{2(2 \pi)^{\frac{1}{2}} \sin ^{2} \beta} \frac{\sin (\varphi / 2)}{\cos ^{2}(\varphi / 2)} .
$$

This result was also obtained by Keller by expanding the exact solution of a special half-plane diffraction problem. It is easily seen that our results agree with those given by Keller in Ref. $1 .^{18}$

In order to complete our general treatment of diffraction by a plane screen, it is necessary to verify that the conditions (4.5a) and (4.5b) are satisfied. These conditions result from imposing the boundary condition (2.16a) and (2.16b) on the asymptotic solution [(4.1) and (4.3)]. First we show that

$$
\begin{array}{r}
\hat{z}_{m}^{\mathrm{r}}\left(x_{1}, x_{2}, x_{3}\right)= \pm \hat{z}_{m}^{\mathrm{i}}\left(x_{1}, x_{2},-x_{3}\right), \\
m=0,1,2, \cdots .
\end{array}
$$

From (5.4) and (5.5) it is clear that the points with rectangular coordinates $\left(x_{1}, x_{2}, \pm x_{3}\right)$ will have ray coordinates $(\sigma, \pm \varphi, \eta)$. Hence it follows from (4.17) and (4.18) that (5.38) is valid for $m=0$. The validity of (5.38) for arbitrary $m$ can then be established by an induction argument. Secondly we state that

$$
\begin{gathered}
\hat{z}_{m}^{\mathrm{i}}\left(x_{1}, x_{2},+0\right)=-\hat{z}_{m}^{\mathrm{i}}\left(x_{1}, x_{2},-0\right), \\
\frac{\partial \hat{z}_{m}^{\mathrm{i}}}{\partial n}\left(x_{1}, x_{2},+0\right)=-\frac{\partial \hat{z}_{m}^{\mathrm{i}}}{\partial n}\left(x_{1}, x_{2},-0\right) .
\end{gathered}
$$

To prove this we extend the domain of definition of the functions $\hat{z}_{m}^{\mathrm{i}}(\sigma, \varphi, \eta)$ to arbitrary values of $\varphi$. (Only the interval $-\pi \leq \varphi \leq \pi$ corresponds to physical space.) Then it follows from (4.17) that

$$
\hat{z}_{m}^{i}(\sigma, \varphi+2 \pi, \eta)=-\hat{z}_{m}^{i}(\sigma, \varphi, \eta)
$$

for $m=0$, and (5.40) can be established for $m=0$, $1,2, \cdots$, by another induction argument. If we now take $\varphi=-\pi$, we find that

$$
\begin{gathered}
\hat{z}_{m}^{\mathrm{i}}(\sigma, \pi, \eta)=-\hat{z}_{m}^{\mathrm{i}}(\sigma,-\pi, \eta), \\
\frac{\partial \hat{z}_{m}^{\mathrm{i}}}{\partial \varphi}(\sigma, \pi, \eta)=-\frac{\partial \hat{z}_{m}^{\mathrm{i}}}{\partial \varphi}(\sigma,-\pi, \eta),
\end{gathered}
$$

${ }^{18}$ We must first correct an error in the last part of Eq. (12) of Ref. 1 which has the wrong sign. Then the results agree because $\varphi=\theta-\pi / 2$. Note that here $D^{\prime}$ has been defined so that it is dimensionless. which is equivalent to (5.39). Finally (5.38) and (5.39) imply (4.5a) and $(4.5 \mathrm{~b})$ because $\partial \hat{s} / \partial n$ vanishes on the screen.

\section{APPENDIX A: A SPECIAL FUNCTION}

Let

$$
f(x)=\pi^{-\frac{1}{2}} e^{-i \pi / 4} e^{-i x^{2}} \int_{-\infty}^{x} e^{i t^{2}} d t .
$$

This is an entire function. It is closely related to the Fresnel integral functions. For large real values of $x$, its asymptotic expansion is

$$
\begin{array}{r}
f(x) \sim e^{-i x^{2}} \eta(x)-\frac{1}{2} \pi^{-\frac{1}{2}} e^{i \pi / 4} x^{-1} \sum_{n=0}^{\infty}\left(\frac{1}{2}\right)_{n}\left(i x^{2}\right)^{-n}, \\
x \rightarrow \pm \infty,
\end{array}
$$

where

$$
\begin{aligned}
\left(\frac{1}{2}\right)_{0}=1, \quad\left(\frac{1}{2}\right)_{n} & =\frac{1}{2}\left(\frac{1}{2}+1\right) \cdots\left(\frac{1}{2}+n-1\right), \\
n & =1,2,3, \cdots,
\end{aligned}
$$

and $\eta(x)$ is the unit step function. Thus $\eta(x)=1$ for $x>0$ and $\eta(x)=0$ for $x<0$.

\section{APPENDIX B: PROOF OF LEMMA 3}

From (4.9) and (4.10) we see that the unit vector in the direction of the incident (reflected) ray is

$\mathbf{U}_{1}=\nabla s^{\mathrm{i}, \mathbf{r}}=\cos \beta \mathbf{t}_{1}-\sin \beta \cos \varphi_{0} \mathbf{t}_{2}$

Let

$$
\mp \sin \beta \sin \varphi_{0} \mathbf{t}_{\mathbf{3}} \text {. }
$$

and

$$
\mathbf{U}_{2}= \pm \sin \varphi_{0} \mathbf{t}_{2}-\cos \varphi_{0} \mathbf{t}_{3}
$$

$\mathbf{U}_{3}=\mathbf{U}_{1} \times \mathbf{U}_{2}=\sin \beta \mathbf{t}_{1}+\cos \beta \cos \varphi_{0} \mathbf{t}_{2}$

$$
\pm \cos \beta \sin \varphi_{0} \mathbf{t}_{\mathbf{3}} \text {. }
$$

From (4.12) we have the unit vector in the direction of the diffracted ray:

$$
\mathbf{U}=\cos \beta \mathbf{t}_{1}+\sin \beta \cos \varphi \mathbf{t}_{2}-\sin \beta \sin \varphi \mathbf{t}_{3} .
$$

Let $\varphi=\mp \varphi_{0} \pm \pi \mp \zeta$. Then, for small $\zeta$,

$$
\cos \varphi=-\cos \varphi_{0}+\zeta \sin \varphi_{0}+\frac{1}{2} \zeta^{2} \cos \varphi_{0}+O\left(\zeta^{3}\right),
$$

$\sin \varphi= \pm\left(\sin \varphi_{0}+\zeta \cos \varphi_{0}-\frac{1}{2} \zeta^{2} \sin \varphi_{0}\right)+O\left(\zeta^{3}\right)$,

and

$$
\begin{aligned}
\mathbf{U}= & \mathbf{U}_{1} \pm \zeta \sin \beta \mathbf{U}_{2} \\
& +\frac{1}{2} \zeta^{2} \sin \beta\left[-\sin \beta \mathbf{U}_{1}+\cos \beta \mathbf{U}_{3}\right]+O\left(\zeta^{3}\right) .
\end{aligned}
$$

Let $\mathbf{Q}$ be a point on the edge and let $\mathbf{P}$ and $\hat{\mathbf{P}}$ be points on the incident (reflected) and diffracted rays emanating from $\mathbf{Q}$ at a distance $\sigma$ from $\mathbf{Q}$. Then

$$
\mathbf{P}=\mathbf{Q}+\sigma \mathbf{U}_{1}, \quad \hat{\mathbf{P}}=\mathbf{Q}+\sigma \mathbf{U} .
$$


In a Cartesian coordinate system with basis vectors $\mathrm{U}_{1}, \mathrm{U}_{2}, \mathrm{U}_{3}$,

$$
\begin{aligned}
\mathbf{h}= & \hat{\mathbf{P}}-\mathbf{P}=\sigma\left(\mathbf{U}-\mathbf{U}_{1}\right) \\
= & \sigma\left(-\frac{1}{2} \zeta^{2} \sin ^{2} \beta, \pm \zeta \sin \beta,+\frac{1}{2} \zeta^{2} \sin \beta \cos \beta\right) \\
& +O\left(\zeta^{3}\right) .
\end{aligned}
$$

Hence, for $s=s^{\mathrm{i}, \mathrm{r}}$,

$$
\begin{aligned}
s(\hat{\mathbf{P}})=s(\mathbf{P}+\mathbf{h})= & s(\mathbf{P})+\mathbf{h} \cdot \boldsymbol{\nabla}_{s}(\mathbf{P}) \\
& +\frac{1}{2} \sum_{i, j} h_{i} h_{j} s_{i j}(\mathbf{P})+\cdots,
\end{aligned}
$$

where $s_{i j}=\partial^{2} s / \partial x_{i} \partial x_{j}$. But $s(\mathbf{P})=s^{i}(\mathbf{Q})+\sigma=\hat{s}(\hat{\mathbf{P}})$. Therefore, since $\nabla s=(1,0,0)$,

$$
\hat{s}(\hat{\mathbf{P}})-s(\hat{\mathbf{P}})=\frac{1}{2} \zeta^{2} \sigma \sin ^{2} \beta\left[1-\sigma s_{22}(\mathbf{P})\right]+O\left(\zeta^{3}\right) .
$$

Let $x_{1}=f\left(x_{2}, x_{3}\right)$ be the equation of the incident (reflected) wavefront passing through $\mathbf{P}$. Then $s\left(f, x_{2}, x_{3}\right)=$ const. Therefore

and

$$
s_{1} f_{v}+s_{v}=0, \quad v=2,3
$$

$$
\begin{array}{r}
s_{11} f_{\mu} f_{v}+s_{1 \mu} f_{v}+s_{1} f_{v \mu}+s_{1 v} f_{\mu}+s_{v \mu}=0 \\
v, \mu=2,3 .
\end{array}
$$

Since $(\nabla s)^{2}=s_{1}^{2}+s_{2}^{2}+s_{3}^{2}=1$,

$$
s_{1} s_{1 v}+s_{2} s_{2 v}+s_{3} s_{3 v}=0, \quad v=1,2,3 .
$$

The wavefront can be represented parametrically with parameters $x_{2}, x_{3}$ in the form $\mathbf{x}=\left[f\left(x_{2}, x_{3}\right), x_{2}, x_{3}\right]$. Then $\quad \mathbf{x}_{2}=\left(f_{2}, 1,0\right), \quad \mathbf{x}_{3}=\left(f_{3}, 0,1\right)$, and $\mathbf{x}_{v \mu}=$ $\left(f_{v \mu}, 0,0\right)$. At $\mathbf{P}$, since $\nabla s=\left(s_{1}, s_{2}, s_{3}\right)=(1,0,0)$, we see that $f_{2}=f_{3}=0, s_{11}=s_{12}=s_{13}=0, s_{v \mu}=-f_{v \mu}$ $(\nu, \mu=2,3), \mathbf{x}_{2}=(0,1,0)=\mathbf{U}_{2}, \mathbf{x}_{3}=(0,0,1)=$ $\mathbf{U}_{3}, g_{v \mu}=\mathbf{x}_{v} \cdot \mathbf{x}_{\mu}=\delta_{v \mu}, \mathbf{x}_{1}=\mathbf{x}_{3} \times \mathbf{x}_{2}=(-1,0,0)=$ $-\mathbf{U}_{1}$, and $L_{v \mu}=\mathbf{x}_{1} \cdot \mathbf{x}_{v \mu}=-f_{v \mu}=s_{v \mu}$. It follows that $\rho^{*}=1 / s_{22}=1 / L_{22}$ is the radius of curvature of the normal section in the direction of $\mathbf{U}_{2}$ of the incident (reflected) wavefront at $\mathbf{P}$.

In Fig. 3 we illustrate the vectors $\mathbf{U}_{2}$ and $\mathbf{U}_{3}$ which are tangent to the incident (reflected) wavefront at Q. We also show the angle $\theta$ between these vectors and the principal directions 2 and 3 corresponding to the principal radii of curvature $\rho_{2}$ and $\rho_{3}$ of the wavefront at $\mathbf{Q}$. Since the wavefronts are parallel (i.e., orthog-

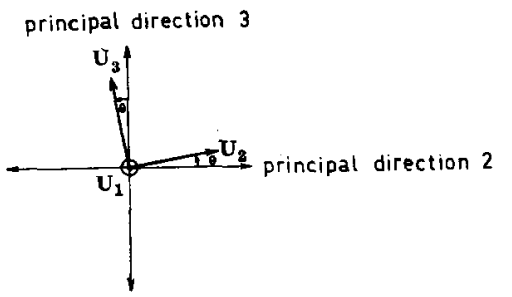

FIG. 3. Principal directions of the incident (reflected) wavefront at $\mathbf{Q}$ (proof of Lemma 3). onal to the same 2-parameter family of rays), the principal radii of curvature of the incident (reflected) wavefront at $\mathbf{P}$ are $\left(\rho_{2}+\sigma\right)$ and $\left(\rho_{3}+\sigma\right)$. Furthermore, the principal directions are the same as those at $\mathbf{Q}$. Therefore, according to Euler's formula,

$$
s_{22}=\frac{1}{\rho^{*}}=\frac{\cos ^{2} \theta}{\rho_{2}+\sigma}+\frac{\sin ^{2} \theta}{\rho_{3}+\sigma} .
$$

From (B1) and (B3) we see that the unit tangent vector to the edge is given by

$$
\mathbf{t}=\mathbf{t}_{\mathbf{1}}=\sin \beta \mathbf{U}_{\mathbf{3}}+\cos \beta \mathbf{U}_{\mathbf{1}} .
$$

In Lemma 2 (Sec. 2) we introduced the radius of curvature $\rho_{0}$ of the normal section of the incident (reflected) wavefront at $\mathbf{Q}$ in the direction of $\mathbf{U}_{3}$ and found that it was equal to $\rho$. Now we see that

$$
\frac{1}{\rho}=\frac{1}{\rho_{0}}=\frac{\sin ^{2} \theta}{\rho_{2}}+\frac{\cos ^{2} \theta}{\rho_{3}} .
$$

If we eliminate $\theta$ from (B15) and (B17), we find that

$$
\begin{aligned}
\left(\rho_{2}+\sigma\right)\left(\rho_{3}\right. & +\sigma) \mathrm{s}_{22} \\
& =\left(\rho_{3}+\sigma\right) \cos ^{2} \theta+\left(\rho_{2}+\sigma\right) \sin ^{2} \theta \\
& =\sigma+\rho_{2}+\rho_{3}-\left(\rho_{2} \cos ^{2} \theta+\rho_{3} \sin ^{2} \theta\right) \\
& =\sigma+\rho_{2}+\rho_{3}-\left(\rho_{2} \rho_{3} / \rho\right) .
\end{aligned}
$$

Hence (B11) becomes

$$
\hat{s}(\hat{\mathbf{P}})-s(\mathbf{P})=\frac{1}{2} \zeta^{2} \sin ^{2} \beta \frac{\sigma \rho_{2} \rho_{3}(\rho+\sigma)}{\rho\left(\rho_{2}+\sigma\right)\left(\rho_{3}+\sigma\right)}+O\left(\zeta^{3}\right) .
$$

Since $z_{0}^{\mathrm{i}, \mathrm{r}}$ is regular at $\mathbf{P}$, we see from (2.8) and (2.9) that

$$
\begin{aligned}
z_{0}^{\mathrm{i}, \mathrm{r}}(\hat{\mathbf{P}}) & =z_{0}^{\mathrm{i}, \mathbf{r}}(\mathbf{P})+O(\zeta) \\
& =z_{0}^{\mathrm{i}, \mathbf{r}}(\mathbf{Q})\left|\frac{\rho_{2} \rho_{3}}{\left(\rho_{2}+\sigma\right)\left(\rho_{3}+\sigma\right)}\right|^{\frac{1}{2}}+O(\zeta)
\end{aligned}
$$

hence

$$
\begin{aligned}
& \frac{\epsilon^{\mathrm{i}, \mathrm{r}}}{2} \pi^{-\frac{1}{2}} e^{i \pi / 4} \frac{z_{0}^{\mathrm{i}, \mathrm{r}}(\hat{\mathbf{P}})}{\sqrt{\hat{s}(\hat{\mathbf{P}})-s(\hat{\mathbf{P}})}} \\
& =\frac{e^{i \pi / 4}}{(2 \pi)^{\frac{1}{2}}} \frac{\epsilon^{i, \mathrm{r} r} z_{0}^{\mathrm{i}, \mathrm{r}}(\mathbf{Q})}{|\zeta| \sin \beta}\left|\frac{\rho}{\sigma(\rho+\sigma)}\right|^{\frac{1}{2}}+O(1) .
\end{aligned}
$$

Since $\varphi=\mp \varphi_{0} \pm \pi \mp \zeta$,

$$
\cos \frac{1}{2}\left(\varphi \pm \varphi_{0}\right)=\frac{1}{2} \zeta+O\left(\zeta^{3}\right) .
$$

Therefore (4.17) and (4.18) yield

$\hat{z}_{\mathbf{0}}^{i, \mathbf{r}}(\hat{\mathbf{P}})=\mp \frac{e^{i \pi / 4}}{(2 \pi)^{\frac{1}{2}}} \frac{z_{0}^{\mathbf{i}, \mathbf{r}}(\mathbf{Q})}{\zeta \sin \beta}\left|\frac{\rho}{\sigma(\rho+\sigma)}\right|^{\frac{1}{2}}+O(\zeta)$

Now $\epsilon^{i, r}= \pm \operatorname{sgn} \zeta$. Therefore $\epsilon^{i, r} /|\zeta|= \pm 1 / \zeta$. According to $(4.6), w_{0}^{\mathrm{i}, \mathrm{r}}(\hat{\mathbf{P}})$ is the sum of $(\mathbf{B} 23)$ and 
(B21); hence $w_{0}^{\mathrm{i}, \mathrm{r}}(\hat{\mathbf{P}})$ has a finite limit as $\zeta \rightarrow 0$, i.e., as $\hat{\mathbf{P}} \rightarrow \mathbf{P}$. It follows that $w_{0}^{\mathrm{i}, \mathrm{r}}(\hat{\mathbf{P}})$ is continuous at the shadow boundary of the incident (reflected) wave.

\section{APPENDIX C: THE LAPLACIAN IN RAY COORDINATES}

We shall transform the Laplacian to the coordinates

$$
\left(y_{1}, y_{2}, y_{3}\right)=(\sigma, \varphi, \eta) \text {, }
$$

which are defined by the transformation (5.4) and (5.5). According to Ref. 16, p. 47,

where

$$
\Delta f=\frac{1}{(g)^{\frac{1}{2}}} \sum_{i=1}^{3} \sum_{j=1}^{3} \frac{\partial}{\partial y_{i}}\left((g)^{\frac{1}{2}} g^{i j} \frac{\partial f}{\partial y_{j}}\right),
$$

and

$$
\begin{aligned}
g_{i j} & =\frac{\partial \mathbf{x}}{\partial y_{i}} \cdot \frac{\partial \mathbf{x}}{\partial y_{j}}, \\
\left(g^{i j}\right) & =\left(g_{i j}\right)^{-1},
\end{aligned}
$$

From (5.4),

$$
g=\operatorname{det}\left(g_{i j}\right)
$$

$$
\frac{\partial \mathbf{x}}{\partial y_{1}}=\mathbf{U}, \frac{\partial \mathbf{x}}{\partial y_{2}}=\sigma \mathbf{U}_{\varphi}, \frac{\partial \mathbf{x}}{\partial y_{3}}=\mathbf{t}_{1}+\sigma \dot{\mathbf{U}} .
$$

Hence

$$
\begin{aligned}
& g_{11}=1, \quad g_{12}=g_{21}=0, \quad g_{13}=g_{31}=\cos \beta, \\
& g_{22}=\sigma^{2} \mathbf{U}_{\varphi}^{2}, \quad g_{23}=g_{32}=\sigma \mathbf{U}_{\varphi} \cdot \mathbf{t}_{1}+\sigma^{2} \mathbf{U}_{\varphi} \cdot \dot{\mathbf{U}}, \\
& g_{33}=1+2 \sigma \mathbf{t}_{1} \cdot \dot{\mathbf{U}}+\sigma^{2} \dot{\mathbf{U}}^{2} .
\end{aligned}
$$

By using (5.5) and (5.3), we may easily obtain $\dot{U}$ and $\mathrm{U}_{\varphi}$. Then, by using (5.6), we find that

$$
\begin{aligned}
& g_{22}=\sigma^{2} \sin ^{2} \beta, \quad g_{23}=\sigma^{2} \tilde{\kappa} \sin \beta \cos \beta \sin \varphi, \\
& g_{33}=1+\frac{2 \sigma \sin ^{2} \beta}{\rho}+\sigma^{2}\left[\left(\frac{\sin \beta}{\rho}\right)^{2}+(\tilde{\kappa} \cos \beta \sin \varphi)^{2}\right] .
\end{aligned}
$$

The determinant (C5) and inverse matrix (C4) may now be computed by standard methods. We find that

and

$$
(g)^{\frac{1}{2}}=\sigma \sin ^{2} \beta\left(1+\frac{\sigma}{\rho}\right),
$$

$$
\begin{aligned}
g g^{11} & =\sigma^{2} \sin ^{2} \beta\left(1+\frac{2 \sigma \sin ^{2} \beta}{\rho}+\frac{\sigma^{2} \sin ^{2} \beta}{\rho^{2}}\right), \\
g g^{12} & =\sigma^{2} \tilde{\kappa} \sin \beta \cos ^{2} \beta \sin \varphi, \\
g g^{13} & =-\sigma^{2} \sin ^{2} \beta \cos \beta, \\
g g^{22} & =\sin ^{2} \beta\left(1+\frac{\sigma}{\rho}\right)^{2}+\sigma^{2} \tilde{\kappa}^{2} \cos ^{2} \beta \sin ^{2} \varphi, \\
g g^{23} & =-\sigma^{2} \tilde{\kappa} \sin \beta \cos \beta \sin \varphi, \quad g g^{33}=\sigma^{2} \sin ^{2} \beta
\end{aligned}
$$

If we set

$$
a^{i j}=(g)^{\frac{1}{2}} g^{i j},
$$

then $(\mathrm{C} 2)$ becomes

$$
\Delta f=\frac{1}{(g)^{\frac{1}{2}}} \sum_{i=1}^{3} \sum_{j=1}^{3}\left[a^{i j} \frac{\partial^{2} f}{\partial y_{i} \partial y_{j}}+\frac{\partial a^{i j}}{\partial y_{i}} \frac{\partial f}{\partial y_{j}}\right] .
$$

Since the $a^{i j}$ are given by (C9)-(C11), (C12) provides a formula for the Laplacian in the coordinates $(\mathrm{Cl})$.

In Sec. 5 we require an expansion of $(\mathrm{C} 12)$ for small $\sigma$. This is easily obtained from our results. We find that

and

$$
\frac{1}{(g)^{\frac{1}{2}}}=\frac{1}{\sigma \sin ^{2} \beta}\left[1-\frac{\sigma}{\rho}+O\left(\sigma^{2}\right)\right]
$$

$$
\begin{aligned}
& a^{11}=\sigma\left[1+\frac{\sigma}{\rho}\left(2 \sin ^{2} \beta-1\right)\right]+O\left(\sigma^{3}\right), \\
& a^{12}=\frac{\sigma \tilde{\kappa} \cos ^{2} \beta \sin \varphi}{\sin \beta}+O\left(\sigma^{2}\right), \\
& a^{13}=-\sigma \cos \beta+O\left(\sigma^{2}\right),
\end{aligned}
$$

$a^{22}=\frac{1}{\sigma}\left(1+\frac{\sigma}{\rho}\right)+O(\sigma), \quad a^{23}=O(\sigma), \quad a^{33}=O(\sigma) ;$ and

$$
\begin{aligned}
& \sum_{i}\left(\frac{\partial a^{i 1}}{\partial y_{i}}\right)=1+\sigma\left[\frac{3 \sin ^{2} \beta-1}{\rho}+\frac{\dot{\beta}}{\sin \beta}\right]+O\left(\sigma^{2}\right), \\
& \sum_{i}\left(\frac{\partial a^{i 2}}{\partial y_{i}}\right)=-\tilde{\kappa} \sin \beta \sin \varphi+O(\sigma), \\
& \sum_{i}\left(\frac{\partial a^{i 3}}{\partial y_{i}}\right)=-\cos \beta+O(\sigma) .
\end{aligned}
$$

By inserting (C13)-(C15) in (C12), we find that

$$
\begin{aligned}
\Delta f= & \frac{1}{\sin ^{2} \beta}\left[1-\frac{2 \cos ^{2} \beta}{\rho} \sigma+O\left(\sigma^{2}\right)\right] f_{\sigma \sigma} \\
& +\left[\frac{2 \tilde{\kappa} \cos ^{2} \beta \sin \varphi}{\sin ^{3} \beta}+O(\sigma)\right] f_{\sigma \varphi} \\
& +\left[\frac{1}{\sigma^{2} \sin ^{2} \beta}+O(1)\right] f_{\varphi \varphi} \\
& +\left[-\frac{2 \cos \beta}{\sin ^{2} \beta}+O(\sigma)\right] f_{\sigma \eta} \\
& +[O(1)] f_{\varphi \eta}+[O(1)] f_{\eta \eta} \\
& +\left[\frac{1}{\sigma \sin ^{2} \beta}+\frac{\tilde{\kappa} \cos \varphi}{\sin ^{3} \beta}-\frac{3 \cos ^{2} \beta}{\rho \sin ^{2} \beta}+O(\sigma)\right] f_{\sigma} \\
& +\left[-\frac{\tilde{\kappa} \sin \varphi}{\sigma \sin \beta}+O(1)\right] f_{\varphi} \\
& +\left[-\frac{\cos \beta}{\sigma \sin { }^{2} \beta}+O(1)\right] f_{\eta} .
\end{aligned}
$$

